\title{
PROBLEMAS DE DIMENSIONAMENTO DE LOTES COM APLICAÇÕES NUMA FUNDIÇÃO AUTOMATIZADA
}

\author{
Elisangela dos Santos Meza
}

Orientador: Prof. Dr. Marcos Nereu Arenales

Dissertação apresentada ao Instituto de Ciências Matemática de São Carlos, da

Universidade de São Paulo, como parte dos requisitos para obtenção do título de Mestre em Ciências - Área: "Ciências de Computação e Matemática Computacional".

São Carlos - SP

Janeiro de 1997 
Ao Moisés 


\section{AGRADECIMENTOS}

Agradeço ao meu orientador Prof. Dr. Marcos Nereu Arenales pelo apoio, incentivo durante o desenvolvimento deste trabalho e muito pela boa convivência e. amizade.

Aos professores: Murilo Francisco Tomé, José Francisco Ferreira Ribeiro, Cassilda Maria Ribeiro e Marcos Nereu Arenales, com os quais tive oportunidade de me aperfeiçoar através das disciplinas cursadas.

Agradeço a Beth, Laura e Marilia da secretaria da pós-gradução, a Sandra da seção de bolsas pela atenção e o bom atendimento, as bibliotecárias pela disponibilidade, e a todos os funcionários de maneira geral.

A todas as pessoas que conheci e convivi durante o mestrado pela amizade, entre elas, Célia, Maristela, Cris, Janaina, Marcos, Robinson, Renato, Alessandro, Luciane Grossi e Lauro.

As meninas da sala 142: Maristela (maristica) e Cris (cris cris) pela amizade, boa convivência e principalmente pelas muitas risadas.

A Janaína e ao Flavio (IME-USP) que contribuíram para o desenvolvimento deste trabalho.

Agradeço a FAPESP e ao CNPq pelo apoio financeiro.

Agradeço aos meus pais, Inez e Waldemar, pela compreensão e amor e, a minha mãe pelas palavras de incentivo e presença constante. A minha irmã Lisi pela amizade e carinho.

Agradeço ao meu marido e companheiro, Moisés, pelo amor, carinho, paciência e compreensão nas horas mais difíceis e muito pelo incentivo.

Agradeço a Deus por ter me dado oportunidade e sabedoria para poder virar mais uma página no livro de minha vida. 


\section{RESUMO}

Neste trabalho estudam-se problemas de dimensionamento de lotes com aplicações específicas em uma fundição automatizada. Quando várias ligas são consideradas na programação, o problema é modelado com um problema de programação linear inteira mista; caso contrário como um problema de programação linear. Algumas hipóteses são assumidas sobre o gargalo e heurísticas são desenvolvidas. Mostra-se também como a fundição pode ser integrada a um sistema de múltiplos estágios, tais como usinagem e montagem. Experiências computacionais sobre o desempenho dos métodos são relatadas. 


\section{ABSTRACT}

In this work the subject of lot sizing problems is studied and a case study in an automated cast is presented. When different alloys are considered in the production scheduling, the problem is modeled as a linear mixed-integer programming problem; otherwise a linear programming problem is used. Some hypotheses on the production bottleneck are assumed and heuristic methods are developed. It is also shown how the automated cast can be coupled with a multilevel global system. Computational performance of the methods is verified and the results presented. 


\section{ÍNDICE}

INTRODUÇÃO. .1

CAPÍTULO 1 Modelos matemáticos para problemas de dimensionamento de lotes.............................................4

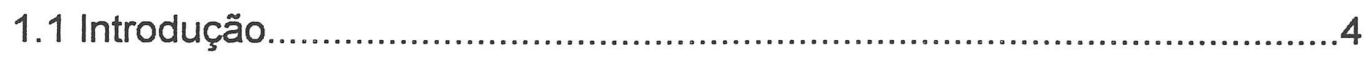

1.2 Problemas de dimensionamento de lotes...............................................

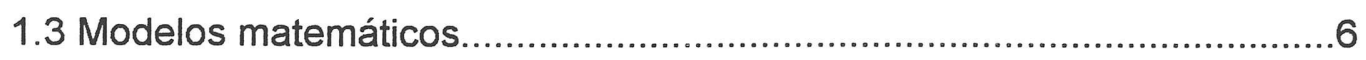

1.3.1 Problemas de dimensionamento de lotes monoestágio......................6

1.3.2 Problemas de dimensionamento de lotes com múltiplos estágios......9

\section{CAPÍTULO 2 Programação da produção numa fundição automatizada}

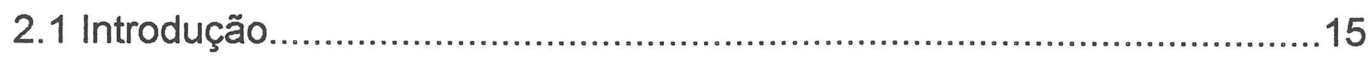

2.20 problema da fundição com uma liģa....................................................17

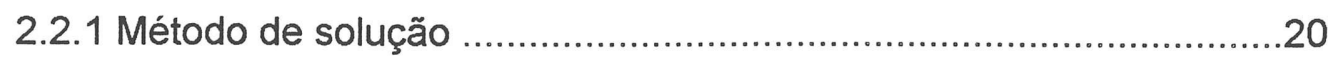

2.2.2 Algoritmo 
2.2.3 Experiências computacionais.

$2.3 \bigcirc$ problema da fundição com várias ligas..........................................26

2.3.1 Método de solução ...........................................................27

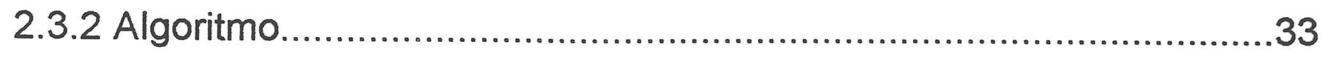

2.3.3 Experiências computacionais....................................................34

CAPÍTULO 3 Um problema de dimensionamento de lotes com múltiplos estágios e com limitações de capacidade...36

3.1 Introdução .36

3.2 Formulação matemática do problema de dimensionamento de lotes com múltiplos estágios e limitações de capacidade.

3.2 Integração do estágio da fundição com os estágios da usinagem e montagem. .42

3.4 Método de solução .43

APÊNDICE 1 


\section{INTRODUÇÃO}

Segundo Shapiro [34], a programação matemática é um rico formalismo com o qual modelos de otimização, para o planejamento e a programação da produção, podem ser construídos e resolvidos. Esses modelos fornecem os detalhes da produção com o objetivo de analisar os problemas mais globais da indústria e desenvolver aproximações eficazes para coordenar ou integrar uma ampla cadeia de atividades de produção. Desta forma, colaboram para incrementar as receitas ou reduzir os custos.

Os problemas de planejamento e programação da produção podem ser modelados como problemas de programação linear, não linear, linear inteira mista, programação dinâmica, estocástica, ou fluxo em redes, dependendo das decisões que são consideradas importantes e que devem ser tomadas.

Ultimamente, os administradores de empresas têm mostrado maior interesse na utilização da programação matemática. Este interesse é devido, em grande parte, às vantagens tecnológicas existentes que permitem aos gerentes adquirirem e organizarem os dados de produção numa base oportuna. Assim, estes sistemas de decisões suportes, baseados em modelos, podem auxiliar na tomada de decisões.

Entre os problemas de planejamento e programação da produção encontrase o problema de dimensionamento de lotes que tem por objetivo determinar quanto (tamanho do lote) deve ser produzido de cada item, final ou intermediário, em cada período, com a finalidade de atender a demanda e otimizar um objetivo (como por exemplo, minimizar os custos, maximizar os lucros ). 
O objetivo deste trabalho é, numa primeira fase, modelar e resolver duas modificações para um problema de programação da produção numa fundição automatizada, anteriormente estudado por Vianna e Arenales [42] e, numa segunda fase, integrar estes problemas com um outro problema estudado por Santos [35], que pode representar os estágios da usinagem e montagem de uma indústria.

Na primeira modificação, foi considerado que com o uso de uma única liga são produzidas todas as peças da fundição. Deste modo, as decisões a serem tomadas são quanto, quando e onde (em que máquina) cada peça deverá ser produzida com a finalidade de atender as demandas das peças em cada período do horizonte de planejamento minimizando os custos de produção. Mesmo sendo um programa linear, foi proposto um método ótimo que explora a estrutura da matriz de restrições, cuja característica é esparsa, para o caso em que o gargalo de produção é o forno.

$\mathrm{Na}$ segunda modificação, foram consideradas várias ligas, sendo possível, em cada período do horizonte de planejamento, a produção de um único tipo de liga que é destinado à produção de um determinado conjunto de peças. Portanto, tem-se duas decisões importantes e interligadas a serem tomadas que são: decidir qual a liga que será produzida em cada período do horizonte de planejamento e em que máquina serão produzidas as peças a fim de atender as demandas em cada período minimizando os custos de produção. Neste caso, tem-se um problema linear inteiro misto. Para resolvê-lo foi proposto um método heurístico, onde o problema relaxado é um problema de dimensionamento de lotes monoestágio com limitações de capacidade.

Qualquer um destes problemas da fundição pode ser vistos como uma fábrica independente ou como o último estágio de qualquer indústria que necessite de metais fundidos para poder fabricar o produto final. Sendo assim, numa segunda parte é feita a integração do problema da fundição com o problema de dimensionamento de lotẹs com múltiplos estágios e limitações de capacidade, estudado por Santos [35], que neste caso representa os estágios da usinagem e montagem de uma indústria. Esta integração é feita da seguinte maneira: o requerimento do estágio da usinagem é a demanda do estágio da fundição. Desta forma, primeiro é resolvido o problema que representa os estágios da usinagem e montagem e depois é programada a produção da fundição. 
O trabalho está organizado da seguinte forma:

No capítulo 1 é apresentada uma introdução geral aos problemas de dimensionamento de lotes, bem como modelos matemáticos para problemas de dimensionamento de lotes monoestágios e com múltiplos estágios. Também são relacionados alguns trabalhos encontrados na literatura.

No capítulo 2 são apresentados dois modelos para o problema de programação da produção numa fundição automatizada, assim como os métodos de solução para os mesmos.

No capítulo 3 é apresentada a integração do problema da fundição com um problema que representa os estágios da usinagem e montagem da indústria.

São apresentados os comentários finais e conclusões.

E no apêndice 1 é apresentado um exemplo do segundo problema da fundição. 


\section{CAPÍTULO 1}

\section{Problemas de dimensionamento de lotes}

\subsection{Introdução}

Neste capítulo descreve-se problemas de dimensionamento de lotes monoestágio e com múltiplos estágios bem como, exemplos de modelos matemáticos para os mesmos. São descritos também alguns métodos de solução encontrados na literatura.

\subsection{Problema de dimensionamento de lotes}

O problema de dimensionamento de lotes tem por objetivo determinar o tamanho do lote a ser produzido de cada item, final ou intermediário, em cada período, com a finalidade de atender a demanda $\curvearrowright$ otimizar uma função objetivo (por exemplo, minimizar os custos, maximizar os lucros ).

Os problemas de dimensionamento de lotes, segundo Bahl et al. [5], podem ser classificados como visto na figura 1.1. 


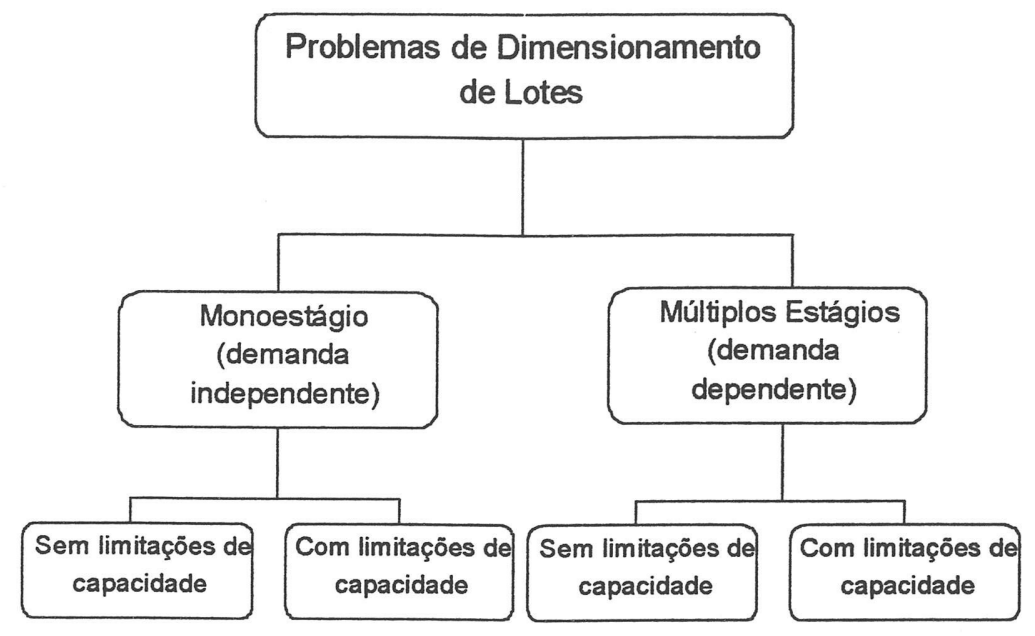

Figura 1.1 Classificação dos problemas de dimensionamento de lotes

Um problema de dimensionamento de lotes monoestágio é um problema de produção onde os itens a serem produzidos são independentes, isto é, nenhum item depende da produção de outro item.

O principal interesse da classe de problemas de dimensionamento de lotes monoestágio sem limitações de capacidade é a determinação dos tamanhos dos lotes de um único item ao longo de vários períodos de tempo, de modo a minimizar os custos envolvidos com a produção do item, satisfazendo uma demanda preestabelecida, Bahl et al. [5].

Um problema de dimensionamento de lotes com múltiplos estágios é um problema de produção, onde um item final possui itens predecessores que também devem ser programados para produção ou compra. Tais itens podem ainda possuir suas próprias demandas chamadas independentes (ou externas).

Billington et al. [9] descreveram quatro tipos de estrutura de produto para problemas de dimensionamento de lotes com múltiplos estágios, ilustrados na figura 1.2. Cada quadrado refere-se a um item a ser produzido ou comprado e cada item é associado com exatamente um estágio na estrutura de produto. Os estágios são enumerados de 0 a L, onde itens no estágio 0 são chamados itens finais, itens no estágio $L$ são chamados matérias-primas e itens nos outros estágio são chamados itens intermediários. Uma linha liga cada item a um item sucessor imediato, o qual necessita-o para poder ser produzido.

A figura 1.2(a) - Serial - ilustra um único item final produzido numa série subsequente de etapas. Cada item, com exceção do primeiro e do último, tem exatamente um sucessor e um predecessor. 
A figura 1.2(b) - Paralelo - ilustra uma estrutura em série com múltiplos itens, onde vários itens passam pelos mesmos estágios de produção.

A figura 1.2(c) - Montagem - representa um produto dado por uma complexa estrutura de montagem. Cada item tem exatamente um sucessor podendo ter vários predecessores.

A figura 1.2(d) - Geral - representa a situação onde não há restrições quanto ao número de predecessores e sucessores de um item, exceto os itens finais que não possuem sucessores.

(a) Serial

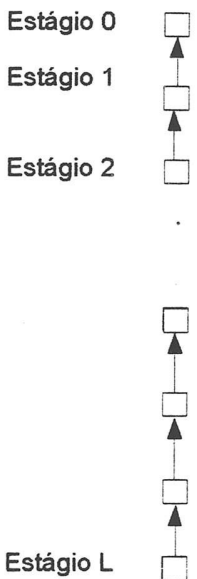

(b) Paralelo

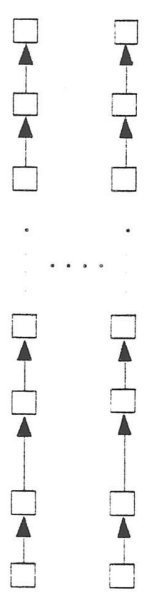

(c) Montagem

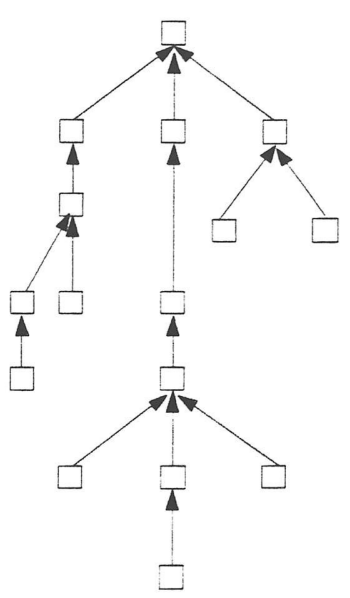

(d) Geral

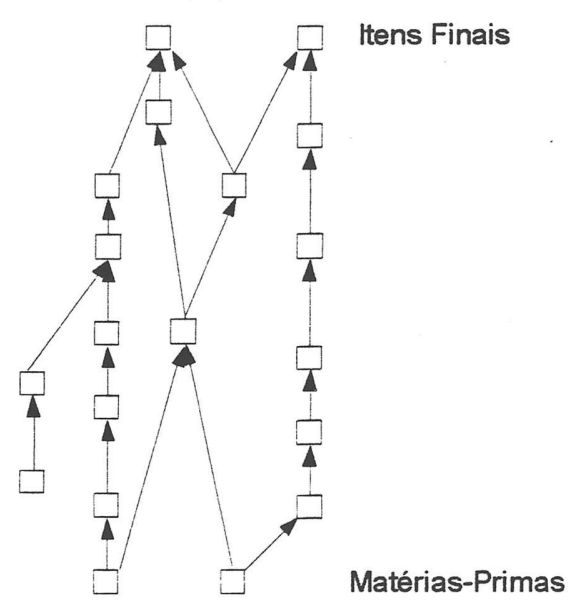

Figura 1.2 Quatro estruturas de produto

\subsection{Modelos matemáticos}

Um problema de dimensionamento de lotes é modelado como um problema linear se o tempo e o custo de preparação (setup) forem irrelevantes e, como um problema linear inteiro misto se o tempo e/ou o custo de preparação forem significativos.

\subsubsection{Problema de dimensionamento de lotes monoestágio}

O problema de dimensionamento de lites monoestágio modelado abaixo encontra-se Trigeiro et al. [38]. Foi considerado a demanda conhecida sobre os $T$ períodos do horizonte de planejamento, a capacidade disponível limitada e a existência de tempo e custo de preparação. 
Foram considerados os seguintes dados:

$d_{i t}$ demanda externa (independente) do item i no período $t$.

$h_{i t} \quad$ custo de estocar uma unidade do item $i$ no período $t$.

$c_{i t} \quad$ custo de produzir uma unidade do item $i$ no período $t$.

$c s_{i t} \quad$ custo de preparação para produzir o item $i$ no período $t$.

$s_{i k} \quad$ tempo de preparação da máquina $k$ para produzir o item $i$.

$b_{i k} \quad$ tempo necessário para produzir uma unidade do item $i$ na máquina $k$.

$C A P_{k t}$ capacidade disponível (em unidades de tempo) da máquina $k$ no período $t$.

q qualquer número grande.

As seguintes variáveis :

$P_{i t} \quad$ quantidade do item $i$ produzida no período $t$.

$I_{\text {it }}$ quantidade do item $i$ estocada no final do período $t$.

$X_{i t}=\left\{\begin{array}{l}1, \text { se existe preparaç ão para produç ão do item i no período } t, \\ 0, \text { em caso contrário. }\end{array}\right.$

E os seguintes índices:

$i=1, \ldots, N \quad$ itens.

$k=1, \ldots, K \quad$ máquinas.

$t=1, \ldots, T \quad$ períodos..

Portanto Trigeiro et al. [38] formularam o problema de dimensionamento de lotes monoestágio como um problema linear inteiro misto da seguinte forma: 
(PM):

Minimize $\sum_{i=1}^{N} \sum_{t=1}^{T}\left(h_{i t} l_{i t}+c_{i t} P_{i t}+c s_{i t} X_{i t}\right)$

Sujeito a:

$$
\begin{aligned}
& I_{i(t-1)}+P_{i t}-I_{i t}=d_{i t} \quad i=1, \ldots, N \quad t=1, \ldots, T \\
& \sum_{i=1}^{N}\left(b_{i k} P_{i t}+s_{i k} X_{i t}\right) \leq C A P_{k t} \quad k=1, \ldots, K \quad t=1, \ldots, T \\
& P_{i t}-q X_{i t} \leq 0 \quad i=1, \ldots, N \quad t=1, \ldots, T \\
& P_{i t} \geq 0 \text { e } I_{i t} \geq 0 \quad X_{i t}=0 \text { ou } 1 \quad i=1, \ldots, N \quad t=1, \ldots, T
\end{aligned}
$$

A função objetivo (1.1) representa os custos de produção, estoque e preparação dos $N$ itens ao longo dos $T$ períodos que se tem por objetivo minimizar. As restrições (1.2) representam o balanceamento de estoque de cada item no final de cada período. As inequações (1.3) são as restrições indicando as limitações de capacidade de cada máquina em cada período e (1.4) são as restrições lógicas indicando que se há produção então há preparação.

Um problema de dimensionamento de lotes monoestágio sem limitações de capacidade e sem custo de preparação é dado por (1.1), (1.2) e (1.5). Se há produção de um único item, isto é, $N=1$, pode-se encontrar a solução ótima para este problema ( (1.1), (1.2) e (1.5)) usando o algoritmo de programação dinâmica proposto por Wagner e Whitin [40]. Se forem considerados vários itens, por exemplo $\mathrm{N}$, o problema pode ser decomposto em $\mathrm{N}$ subproblemas, um para cada item, que também podem ser resolvidos pelo mesmo algoritmo de Wagner e Whitin.

Para resolver o problema monoestágio com limitações de capacidade existem na literatura métodos ótimos, quase ótimos e heurísticos. Dentre os que desenvolveram métodos ótimos/quase ótimos, destacam-se: Diaby et al. [17] que desenvolveram métodos utilizando branch-and-bound no qual, os limitantes foram gerados utilizando a Relaxação Lagrangeana e os custos duais foram atualizados pela Otimização do Subgradiente e, Toledo et al. [37] que representaram o 
problema como uma rede generalizada e desenvolveram um método ótimo. $\mathrm{O}$ método é um branch-and-bound especializado, onde em cada nó é resolvido um problema de custo mínimo na rede generalizada.

Dentre os métodos heurísticos desenvolvidos destacam-se: o método heurístico proposto por Trigeiro et al. [38] que consiste em relaxar as restrições de capacidade, aplicando a Relaxação Lagrangeana, obtendo deste modo $\mathrm{N}$ subproblemas sem restrições de capacidade os quais foram resolvidos pelo algoritmo de Wagner e Whitin. Em seguida, é verificado se a solução é infactivel. Em caso afirmativo, é aplicado um método de suavização que transfere produção entre períodos, na tentativa de factibilizar a solução e, finalmente os multiplicadores duais são atualizados utilizando o método de Otimização do Subgradiente. Outros métodos heurísticos são encontrados em Dixon e Silver [18], Lembrechet e Venderveken [25] e Maes e Van Wassenhove [29].

Nos métodos de solução propostos no capítulo 2, o problema relaxado é um problema de dimensionamento de lotes monoestágio com limitações de capacidade sem tempo e custo de preparação, portanto um programa linear.

\subsubsection{Problema de dimensionamento de lotes com múltiplos estágios}

A diferença de um problema de dimensionamento de lotes monoestágio para um com múltiplos estágios, como visto anteriormente, está na dependência entre os itens. Num problema monoestágio os itens são produzidos independentemente. Já num problema com múltiplos estágios, a produção de um item depende da produção de um ou mais itens que o precedem, ou de outra forma, a produção de um item gera uma demanda de outros itens. Para exemplificar considere a figura 1.3.

Observe que os itens são enumerados de forma que se o item $j$ for sucessor do item $i$ então $j<i, \forall i$ e $\forall j$. Define-se $a_{i j}$ como a quantidade do item $i$ necessária para produzir uma unidade do item $j$, (note que, $a_{i j}$ existe apenas para os itens $j$ sucessores imediatos do item $i$ ). Consequentemente, $\sum_{j} a_{i j} P_{j}$ (onde $P_{j}$ é a produção do item $j$ ) é a demanda gerada ou demanda dependente do item $i$. 


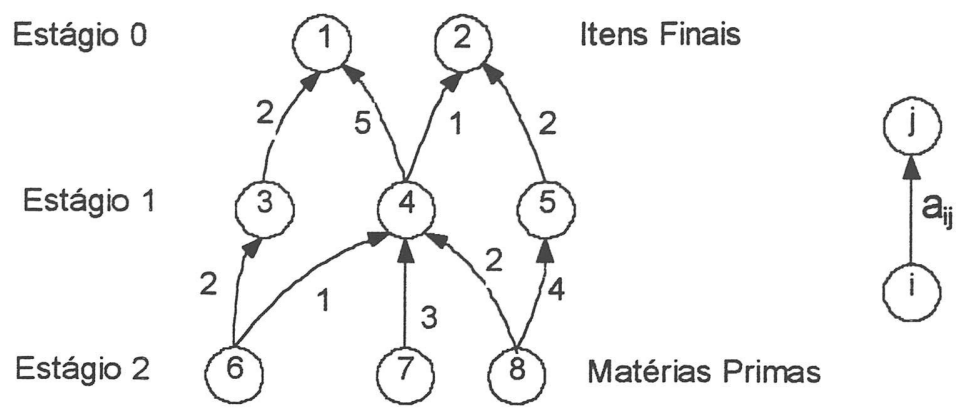

Figura 1.3 Uma estrutura geral de produto

Note que os predecessores imediatos, por exemplo, do item 4 são os itens $\{6,7,8\}$ e os sucessores imediatos do item 4 são os itens $\{1,2\}$. Suponha, por exemplo, que se deseja produzir $P_{1}$ unidades do item 1 e $P_{2}$ unidades do item 2; do estágio 0 , (itens finais), assim a demanda dependente do item 4 , do estágio 1 , é $5 P_{1}+P_{2}$. Isto caracteriza um problema com múltiplos estágios.

Billington et al. [9] formularam um problema de dimensionamento de lotes com múltiplos estágios e limitações de capacidade, como um problema linear inteiro misto.

Os dados adicionais ao problema de dimensionamento de lotes monoestágio (PM) são:

$L_{i} \quad$ lead time do item $i$.

$y_{i} \quad$ fração média de aproveitamento da produção do item $i$.

$a_{i j} \quad$ quantidade do item $i$ necessária para produzir uma unidade do item $j$, ( $j$ sucessor imediato do item $i$ ).

Observe que os dois primeiros dados podem ser incorporados ao modelo monoestágio.

E os seguintes índlces:

$i=1, \ldots, M \quad$ itens finais.

$i=M+1, \ldots, N$ itens intermediários. 
O modelo matemático é o seguinte:

(PME):

Minimize $\sum_{i=1}^{N} \sum_{t=1}^{T}\left(h_{i t} l_{i t}+c_{i t} P_{i t}+c s_{i t} X_{i t}\right)$

Sujeito a:

$$
\begin{aligned}
& l_{i(t-1)}+y_{i} P_{i\left(t-L_{i}\right)}-I_{i t}-\sum_{j=1}^{N} a_{i j} P_{j t}=d_{i t} \quad i=1, \ldots, N \quad t=1, \ldots, T \\
& \sum_{i=1}^{N}\left(b_{i k} P_{i t}+s_{i k} X_{i t}\right) \leq \mathrm{CAP} P_{t k} \quad k=1, \ldots, K \quad t=1, \ldots, T \\
& P_{i t} \leq q X_{i t} \quad i=1, \ldots, N \quad t=1, \ldots, T \\
& P_{i t} \geq 0 \text { e } l_{i t} \geq 0, X_{i t}=0 \text { ou } 1 \quad i=1, \ldots, N \quad t=1, \ldots, T
\end{aligned}
$$

O modelo (1.6)-(1.10) é igual ao problema (1.1)-(1.5), a menos das restrições de balanceamento de estoque (1.7) que consideram a demanda dependente, $\sum_{j=1}^{N} a_{i j} P_{j t}$, que caracteriza , como mencionado acima, o problema de dimensionamento de lotes com múltiplos estágios. Também foi considerado o lead time, $L_{i}$. Lead time é o tempo inevitável desde o momento em que um produto é encomendado até estar disponível. Este pode ser o tempo que um fornecedor demora para entregar um produto ou tempo não produtivo, tal como o tempo para a pintura secar, o metal quente esfriar, ou um lote ser movido fisicamente entre duas áreas de produção (Billington et al. [9]).

Para o problema com múltiplos estágios, uma maneira de torná-lo decomponível em $\mathrm{N}$ subproblemas, como no caso monoestágio, é reformulá-lo em termos de estoque de escalão, ver Berreta [8]. 
Há várias pesquisas sobre métodos de solução para o problema de dimensionamento de lotes com múltiplos estágios considerando variações na estrutura de produto e/ou nas limitações de capacidade.

Um problema de dimensionamento de lotes com múltiplos estágios e sem limitações de capacidade é dado a partir do problema (PME) omitindo as restrições (1.8).

Este problema foi estudado por Afentakis et al. [2], que propuseram um algoritmo branch-and-bound para a estrutura de montagem e, Afentakis e Gavish [1] estudaram o problema considerando a estrutura geral de produto mas, transformaram o problema para uma estrutura equivalente de montagem e aplicaram o mesmo método dado em Afentakis et al. [2]. Métodos heurísticos para esse problema são encontrados em Kuik e Salomon [23] e Blackburn e Millen [12].

Dentre os que estudaram o problema de dimensionamento de lotes com múltiplos estágios e limitações de capacidade destacam-se: Billington et al. [9] que apresentaram um método de redução do problema (PME), mas não apresentaram um método de solução. Billington et al.[10] que consideraram o modelo (PME) com restrições de capacidade em apenas um estágio na estrutura geral de produto, isto é, consideraram um único centro de trabalho gargalo. (Um gargalo é um centro de trabalho que limita a taxa de produção de toda a fábrica). O método de solução proposto é um branch-and-bound com heurísticas. Em qualquer nó da árvore de ramificação, um problema restrito foi definido com valores fixos para algumas variáveis $X_{i t}$ (variáveis de preparação) e, para cada item $\mathrm{N}$ foi feita uma relaxação Lagrangeana heurística, isto é, foram relaxadas todas as restrições de capacidade (relacionadas ao centro de trabalho gargalo) e todas as restrições de balanceamento de estoque, exceto $T$ restrições (uma para cada período) de um item selecionado. Esses $\mathrm{N}$ subproblemas são resolvidos a partir dos itens finais às matérias primas e de estágio a estágio de forma que quando se programa a produção de um item a demanda dependente para o mesmo é conhecida, portanto cada subproblema é na. verdade um problema monoestágio sem limitações de recursos e pode ser resolvido pelo algoritmo de Wagner e Whitin. Após, um método de suavização é aplicado para cijustar a produção dentro da capacidade disponível, se possível produzindo uma solução factível. Em seguida os custos duais são atualizados para desestimular a produção em períodos com produção excessiva, pelo método de Otimização do Subgradiente. 
Billington et al. [11] estudaram o desempenho de várias heurísticas desenvolvidas para o problema com múltiplos estágios. Kuik et al. [24], consideraram o problema com restrições de capacidade em apenas um estágio como em Billington et al. [10], não consideraram tempo de preparação, lead time nem estrutura geral. Estudaram três técnicas heurísticas, uma baseada em programação linear e, outras duas baseadas em técnicas de buscas heurísticas.

Maes et al. [28] apresentaram uma heurística para resolver o problema com limitações de capacidade em mais de um estágio. Ignoraram as variáveis inteiras, resolveram o problema linear e em seguida testaram três técnicas heurísticas para "arredondar" as variáveis inteiras.

Clark e Armentano [15] desenvolveram uma heurística para resolver o problema com limitações de capacidade para a estrutura geral de produto considerando lead time diferente de zero e, tempo e custo de preparação. 0 problema foi reformulado em termos de estoque de escalão com o intuito de facilitar o funcionamento da heurística proposta. A heurística desenvolvida primeiro obtém uma solução razoável para o problema sem limitações de capacidade e depois tenta obter a factibilidade da solução através da transferência de produção para trás no tempo (método de suavização).

Berreta e Armentano [7] resolveram o problema considerando tempo e custo de preparação, estrutura geral de produto e, a capacidade limitada. Desenvolveram um método heurístico utilizando a formulação do problema em termos de estoque de escalão, como em Clark e Armentano[15]. Inicialmente resolveram o problema sem considerar as restrições de capacidade, em seguida, a heurística tenta factibilizar a solução movendo a produção para frente ou para trás no tempo. Técnicas de busca tabu foram incorporadas à heurística.

Se o tempo e o custo de preparação são irrelevantes, o problema de dimensionamento de lotes com múltiplos estágios com limitações de capacidade pode ser modelado como um programa linear. A programação linear trata facilmente de problemas com restrições de capacidade, ou seja, pode-se determinar um programa ótimo considerando todos os centros restritos.

Afentakis et al. [2] reformularam o problema com estrutura de montagem sem restrições de capacidade em termos de estoque de escalão o que simplifica sua decomposição pela Relaxação Lagrangeana. Desenvolveram um algoritmo branch-and-bound onde os limitantes foram gerados pela resolução do problema 
Lagrangeano. Em Afentakis e Gavish [1], converteu-se a formulação do problema com estrutura geral para uma estrutura de montagem com restrições adicionais mas, esta transformação aumentou significativamente o número de variáveis. Resolveram o problema transformado pelo mesmo método apresentado em Afentakis et al. [2].

McClain et al. [30] consideraram o problema linear e apresentaram um método de decomposição que explora a estrutura especial do programa linear quando os estoques iniciais são nulos. O método consiste em dualizar as restrições de capacidade, obtendo desta forma, um programa linear cuja matriz das restrições constitui uma estrutura de Leontief. Esse problema foi resolvido através de um algoritmo de dois passos.

Santos et al. [36] propuseram um método de solução para um problema de dimensionamento de lotes com múltiplos estágios e com limitações de capacidade, programa linear, que em certos aspectos estende o modelo proposto por Billington et al. [9]. O método proposto baseia-se na Relaxação Lagrangeana. Um grupo de restrições é relaxado obtendo-se um problema que pode ser decomposto em dois subproblemas independentes, os quais são resolvidos analiticamente.

O problema descrito no capítulo 2, problema da fundição, pode ser visto como o estágio predecessor do problema proposto por Santos [35], já que este pode representar os estágios da usinagem e montagem de uma indústria. 


\section{CAPÍTULO 2}

\section{Programação da produção numa fundição automatizada}

\subsection{Introdução}

Segundo Resende [32] os processos de uma fundição consistem em fabricar moldes, preparar e fundir metais, vazar o metal dentro do molde, limpar as peças fundidas e recuperar a areia para a reutilização. O produto da fundição é uma peça que pode variar consideravelmente de peso, como também de composição, isto é, diferentes metais podem ser fundidos.

Os equipamentos de uma fundição são basicamente um forno de fusão, um conjunto de máquinas de.moldagem e um conjunto de máquinas de preparação de areia.

A figura 2.1 fornece uina representação esquemática e simplificada das principais atividades de uma fundição em areia. 


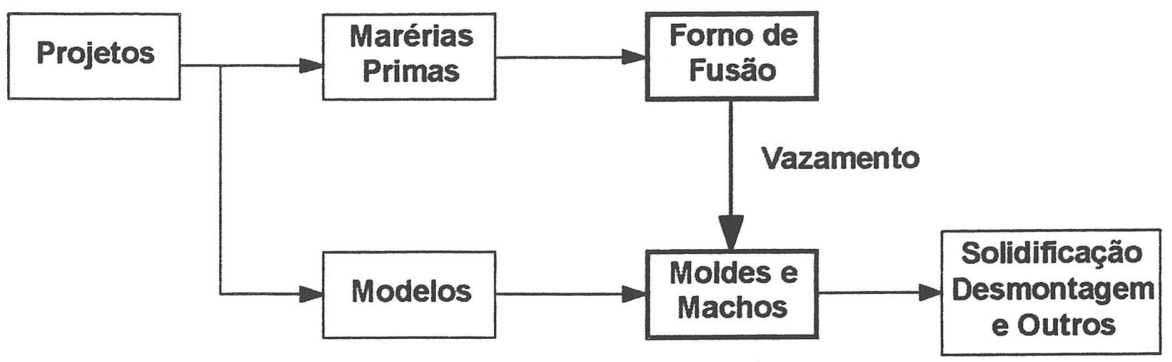

Figura 2.1 Principais atividades numa fundição

O forno é alimentado por matérias-primas (em geral, lingotes de fundidos obtidos após um processo de refino de minérios, sucatas e outros) que produzem um tipo de liga e tem uma capacidade máxima de produção em toneladas por hora, isto é, a cada hora (ciclo do forno) o forno pode produzir uma quantidade máxima. de uma certa liga e, uma vez terminado o vazamento da liga, é então realimentado para a produção de um novo tipo (ou mesmo) de liga. A operação de preparação do forno não foi considerada para os problemas em estudo.

A liga produzida pelo forno é vazada em moldes de areia, os quais são preparados segundo projetos que especificam todos os detalhes dos contornos, canais de vazamento, etc., bem como o tipo de liga adequada. Os moldes de areia, por sua vez, são produtos de máquinas de moldagem que têm capacidades de produção também conhecidas, isto é, é conhecida a quantidade máxima de moldes por hora, para cada máquina, para cada tipo de peça. Essas máquinas de moldagem podem trabalhar uma parte da hora na produção de um tipo de molde e outras partes na produção de outros tipos. Para o caso em estudo, os tempos de preparação dessas máquinas são irrelevantes.

É interessante observar ainda que, embora as máquinas de moldagem possam ser repreparadas para a produção de diferentes moldes dentro de uma hora, ou de um periodo, a liga produzida pelo forno somente pode ser alterada (se for o caso) na mudança de períodos, os quais podem ser de várias horas (esta é a razão de não se considerar a preparação do forno). Portanto, as mudanças na produção de moldes estão restritas às peças que utilizam a mesma liga metálica. Isto mostra que a programação da produção numa fundição, para o caso onde se tem várias ligas, tein dois momentos importantes $e$ interligados que sãc a programação do forno, na qual é definida a liga a ser produzida num determinado período, e a programação das máquinas de moldagem, na qual são definidos os moldes a serem produzidos. 
Em outras fundições podem ser encontradas características diferentes das aqui apresentadas (muitas vezes impostas pela tecnologia instalada para uma variedade de fornos e tipos de moldagens), de modo que as variáveis importantes a serem determinadas, bem como as restrições, podem ser definidas de modo diverso do que aqui considerado e em alguns casos, pode estar representada como mais um estágio no modelo proposto por Santos [35].

Neste capítulo são apresentadas duas reformulações matemáticas e os métodos de solução para um problema de programação da produção numa fundição automatizada, anteriormente estudado por Vianna e Arenales [42]. No problema estudado pelos autores a demanda somente era atendida no final do horizonte de planejamento, isto é, as quantidades de peças em estoque não eram variáveis relevantes. Já nos problemas em estudo há demandas das peças em cada período do horizonte de planejamento e a variável de estoque torna-se uma variável importante na formulação do modelo.

O objetivo destas reformulações, é numa fase posterior, poder integrar o problema de dimensionamento de lotes proposto por Santos [35], modelado para os estágios da usinagem e montagem, com o estágio da fundição. Esta é a situação real de uma indústria.

Deve-se salientar que os métodos de solução a serem apresentados são válidos para o caso em que o forno é o gargalo de produção, ou seja, a capacidade das máquinas deve ser bem maior do que a capacidade do forno em cada período do horizonte de planejamento.

\subsection{O problema da fundição com uma liga}

Neste problema é considerado que com o uso de um único tipo de liga são produzidas todas as peças da fundição. Deste modo, as decisões a serem tomadas são quanto, quando e onde cada peça deverá ser produzida com a finalidade de atender as demandas de $N$ peças ao longo de $T$ períodos do horizonte de planejamento e minimizar cs custos de produção.

São considerados os seguintes dados: 
$a_{i m}$ quantidade da peça $i$ possível de ser produzida na máquina $m$ por hora, $(t n / h)$. Se $a_{i m}=0$ então a peça $i$ não pode ser produzida pela máquina $m$;

$F_{t} \quad$ quantidade máxima de liga produzida pelo forno, por hora, no período $t$, $(\operatorname{tn} / h)$

$h_{t} \quad$ número de horas no período $t,(h)$;

$d_{i t} \quad$ demanda da peça $i$ no período $t(t n /$ período)

$c_{i t} \quad$ custo de produção da peça $i$ no período $t,(\$)$.

As variáveis de decisão são:

$X_{i m t}$ fração de tempo no período $t$ utilizada para produzir peças do tipo i na máquina $m$;

$I_{\text {it }}$ estoque da peça $i$ no final do período $t,(t n)$.

E os índices do problema são:

$m=1, \ldots, M$ máquinas;

$t=1, \ldots, T \quad$ períodos;

$i=1, \ldots, N$ peças.

Pode-se observar que $a_{i m} h_{t} X_{i m t}$ significa a quantidade, em toneladas, de peças do tipo i produzida pela máquina $m$ no período $t$.

Foi formulado o seguinte programa linear: 
Minimize $\sum_{t=1}^{T} \sum_{i=1}^{N} \sum_{m=1}^{M} c_{i t} a_{i m} h_{t} X_{i m t}$

Sujeito a:

$$
\begin{aligned}
& \sum_{m=1}^{M} a_{i m} h_{t} X_{i m t}-I_{i t}+I_{i(t-1)}=d_{i t} \quad i=1, \ldots, N \quad t=1, \ldots T \\
& \sum_{i=1}^{N} \sum_{m=1}^{M} a_{i m} h_{t} X_{i m t} \leq F_{t} h_{t} \quad t=1, \ldots, T \\
& \sum_{i=1}^{N} X_{i m t} \leq 1 \quad t=1, \ldots, T \quad m=1, \ldots, M \\
& X_{i m t} \geq 0 \quad i=1, \ldots, P \quad m=1, \ldots, M \quad t=1, \ldots, T
\end{aligned}
$$

$\mathrm{Na}$ formulação acima a função objetivo (2.1) minimiza o custo total de produção, onde os custos de produção das peças são considerados nãocrescentes ao longo dos períodos (como proposto em Billington et al. [10]), ou seja $c_{i 1} \geq c_{i 2} \geq \ldots . . . \geq c_{i T}$, dessa forma é desestimulado grandes quantidades de peça em estoque entre períodos. (Observe que a introdução de custos de estocagem na função objetivo (2.1) é outra maneira de desestimular grandes quantidades de peças em estoque). As equações (2.2) são as restrições de balanceamento de estoque de cada peça em cada período do horizonte de planejamento; as inequações (2.3) definem a capacidade de produção do forno em cada período $t$, as inequações (2.4) são devido a $X_{i m t}$ ser a fração de tempo e (2.5) são as restrições de não negatividade das variáveis $X_{i m t}$.

Este problema pode ser representado, para um período $t$, como visto na figura 2.2: 


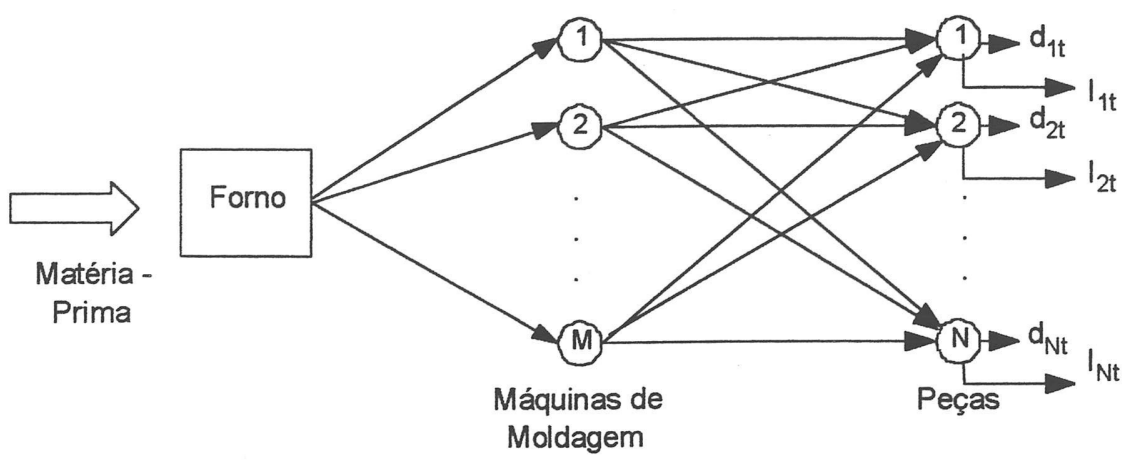

Figura 2.20 problema da fundição com uma liga

\subsubsection{Método de solução}

A matriz de restrições do modelo (2.1) - (2.5) apresenta uma estrutura esparsa particular, conforme a figura 2.3:
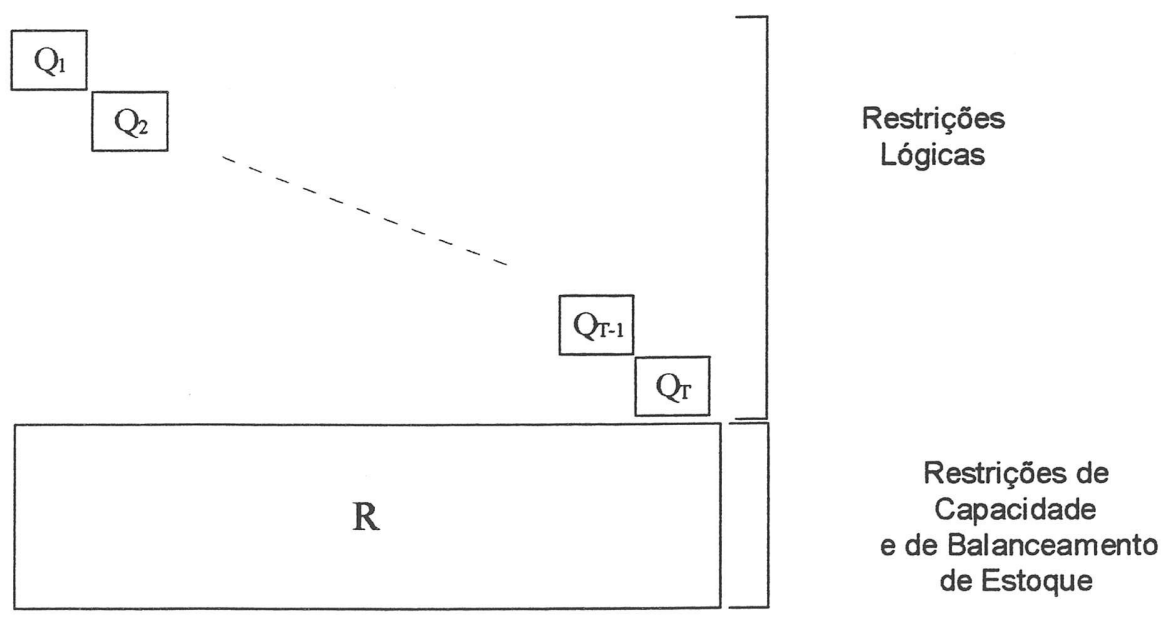

Figura 2.3 Estrutura da matriz dos coeficientes do problema da fundição com uma liga

Onde as submatrizes $Q_{i}$ e $R$ também são esparsas.

Desta forma, mesmo sendo um programa linear, é explorada a estrutura da matriz propondo um método de solução ótimo, para o caso onde o gargalo de produção é o forno. 
Como a capacidade de produção das máquinas foi considerada bem maior do que a capacidade do forno, as restrições (2.4) podem ser relaxadas sem prejuízo da factibilidade da solução, pois, note que, se

$$
\sum_{i=1}^{N} X_{i m t}=2
$$

pode-se definir $X_{i m t}^{\prime}$ como

$$
X_{i m t}^{\prime}=\frac{X_{i m t}}{2}
$$

de modo que

$$
\sum_{i=1}^{N} X_{i m t}^{i}=1 \text { (condição da variável ser fração de tempo) }
$$

e a restrição (2.3) torna-se:

$$
\sum_{i=1}^{N} \sum_{t=1}^{M} 2 a_{i m} h_{t} X_{i m t}^{\prime} \leq F_{t} h_{t}
$$

isto é, tem-se as restrições satisfeitas com as capacidades das máquinas dobradas. Entretanto, a hipótese de folga nas capacidades das máquinas diz que $\sum_{i=1}^{N} X_{i m t}=2$ não pode acontecer (pois isto, como visto, implica em ter que dobrar a capacidade das máquinas para se ter factibilização).

Minimize $\sum_{t=1}^{T} \sum_{i=1}^{N} \sum_{m=1}^{M} c_{i t} a_{i m} h_{t} X_{i m t}$

Sujeito a:

$$
\begin{aligned}
& \sum_{m=1}^{M} \sum_{i=1}^{N} a_{i m} h_{t} X_{i m t} \leq F_{t} h_{t} \quad t=1, \ldots, T \\
& \sum_{m \cdot 1}^{M} a_{i m} h_{t} X_{i m t}-I_{i t}+I_{i(t-1)}=d_{i t} \quad i=1, \ldots, N \quad t=1, \ldots, T \\
& X_{i m t} \geq 0 \quad i=1, \ldots, N, m=1, \ldots, M, t=1, \ldots, T
\end{aligned}
$$


Definindo uma nova variável $P_{i t}$ como:

$$
P_{i t}=\sum_{m=1}^{M} a_{i m} h_{t} X_{i m t}
$$

a quantidade de peças do tipo $i$ produzida no período $t$.

O problema relaxado pode ser reescrito como:

Minimize $\sum_{t=1}^{T} \sum_{i=1}^{N} c_{i t} P_{i t}$

Sujeito a:

$$
\begin{aligned}
& \sum_{t=1}^{N} P_{i t} \leq F_{t} h_{t} \quad t=1, \ldots, T \\
& P_{i t}+I_{i(t-1)}-I_{i t}=d_{i t} \quad t=1, \ldots, T, \quad i=1, \ldots, N \\
& P_{i t} \geq 0 \quad t=1, \ldots, T, \quad i=1, \ldots, N
\end{aligned}
$$

Resolvendo este programa linear que é um problema de dimensionamento de lotes monoestágio capacitado, por um método ótimo apropriado, determina-se $P_{i t}$, a quantidade de cada peça $i$ produzida em cada período $t$.

Este problema pode ser representado esquematicamente como visto na figura 2.4 .

Uma vez resolvido este programa linear, determina-se a programação das máquinas, para cada período $t$ do horizonte de planejamento, obtendo-se a fração de tempo de produção de cada peça em cada máquina. Assim, resolve-se o seguinte problema de transporte generalizado, para cada $t$ fixu. 
Minimize $\sum_{m=1}^{M} \sum_{i=1}^{N} X_{i m t}$

Sujeito a

$$
\begin{aligned}
& \sum_{i=1}^{N} X_{i m t} \leq 1 \quad m=1, \ldots, M \\
& \sum_{m=1}^{M} a_{i m} h_{t} X_{i m t}=P_{i t} \quad i=1, \ldots, N \\
& X_{i m t} \geq 0 \quad m=1, \ldots, M \quad i=1, \ldots, N
\end{aligned}
$$

onde (2.10) minimiza o tempo gasto em cada máquina para a produção das peças no período, (2.11) são devido a $X_{i m t}$ ser a fração de tempo e (2.12) é a definição da variável $P_{i t}$.

Um objetivo alternativo para a programação das máquinas no periodo $t$, é minimizar a máxima utilização das máquinas, isto é:

$$
\operatorname{mim} \max _{m=1, \ldots, M}\left\{\sum_{i=1}^{N} X_{i m t}\right\}
$$

Seja $z_{t}$ uma variável tal que:

$$
z_{t}=\max _{m=1, \ldots, M}\left\{\sum_{i=1}^{N} X_{i m t}\right\} \quad t=1, . ., T
$$

Desta forma pode-se escrever o modelo para a programação das máquinas, para o perícdo $t$ da seguinte forma: 


\section{Minimize $z_{t}$}

Sujeito a

$$
\begin{aligned}
& \sum_{i=1}^{N} X_{i m t} \leq z_{t} \quad m=1, \ldots, M \\
& \sum_{m=1}^{M} a_{i m} h_{t} X_{i m t} \geq P_{i t} \quad i=1, \ldots, N \\
& X_{i m t} \geq 0 \quad m=1, \ldots, M i=1, \ldots, N
\end{aligned}
$$

Observe que este problema é factível se a variável $z_{t} \leq 1$, ou seja, se $z_{t}=\max _{m=1, \ldots, M}\left\{\sum_{i=1}^{N} X_{i m t}\right\} \leq 1$

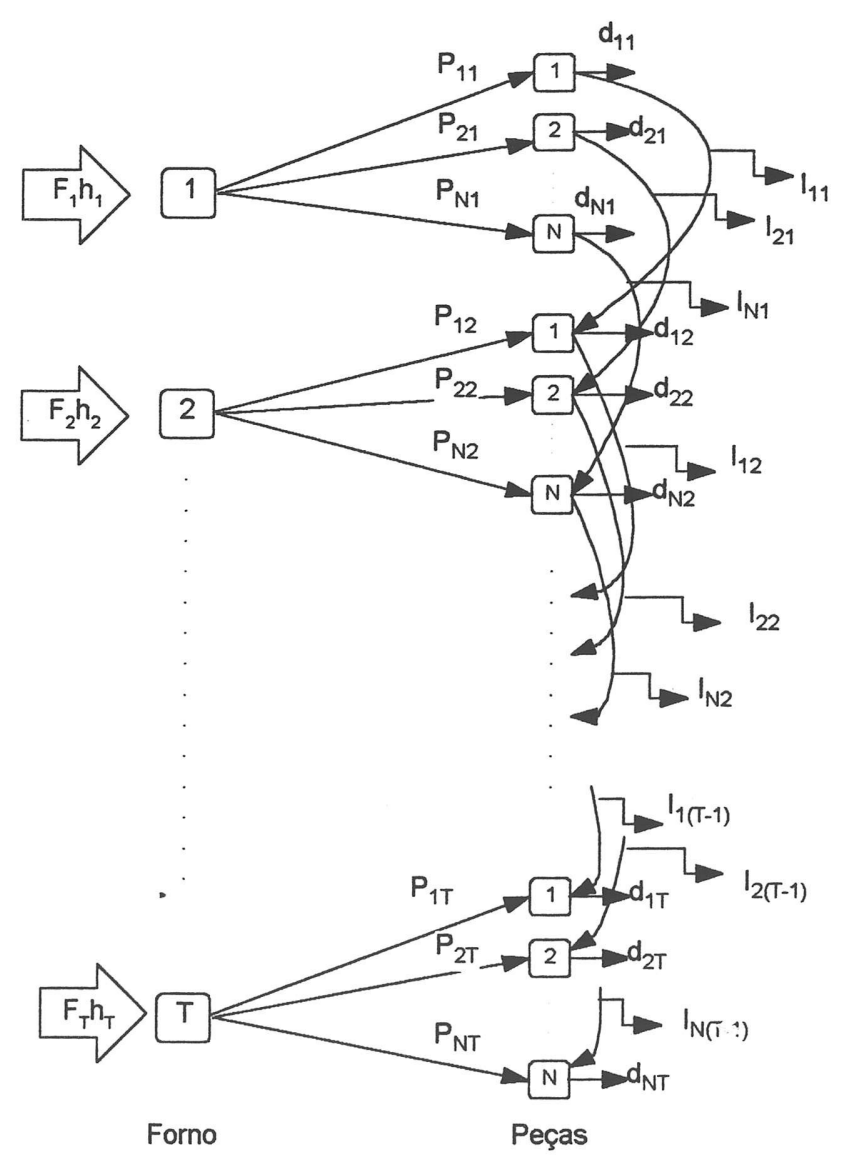

Figura 2.4 Representação do problema relaxado 


\subsubsection{Algoritmo}

- Passo 1

Resolver o problema de dimensionamelnto de lotes monoestágio com limitações de capacidade, modelo (2.6)-(2.9).

- Passo 2

Para cada período $t, t=1, \ldots, T$, resolver (2.10)-(2.13) ou (2.14)-(2.17), determinando $X_{i m t}$, a fração de tempo utilizada para produzir peças do tipo i na máquina $m$. Fim.

\subsubsection{Experiências computacionais}

Para a resolução do algoritmo apresentado na seção 2.2.2, ou seja, para resolver o problema de dimensionamento de lotes monoestágio com limitações de capacidade (2.6)-(2.9) e os problemas (2.10)-(2.13) e (2.14)-(2.17) foi implementado um programa em C que utiliza rotinas do pacote CPLEX versão 3.0. Foram utilizadas as seguintes rotinas deste pacote: para a alocação dos dados foi utilizada a rotina loadlp, para encontrar a solução ótima dos problemas foi utilizada a rotina optimize (que otimiza um problema linear usando o primal simplex), para acessar os valores ótimos da solução dos problemas foi utilizada a rotina solution. $O$ compilador utilizado foi o gcc para estações de trabalho.

Observe que o problema (2.1) - (2.5) tem $\left(N^{*} M^{*} T\right)+(N+T)$ variáveis e $\left(N^{*} T\right)+T+M$ restrições. Já o problema (2.6) - (2.9) tem $2\left(N^{*} T\right)$ variáveis e $\left(N^{*} T+T\right)$ restrições, o problema (2.10) - (2.13) tem $M^{*} N$ variáveis e $M+N$ restrições e o problema (2.14) - (2.17) tem $N^{\star} M+1$ variáveis e $N+M$ restrições.

Em todos os exemplos testados, com a hipótese de que a capacidade do forno é bem menor do que a capacidade das máquinas em cada período do horizonte de planejamento, obteve-se a solução ótima. 


\subsection{O problema da fundição com várias ligas}

Neste problema, são consideradas várias ligas, sendo possível, em cada período do horizonte de planejamento, a produção de um único tipo de liga que é destinado à produção de um determinado conjunto de peças. O objetivo é programar o forno e as máquinas, em cada período, de modo a minimizar o custo de produção atendendo as demandas das peças em cada período do horizonte de planejamento.

Pode ser observado que, no período $t$ poderá existir demandas de peças produzidas por no máximo $t$ ligas, uma vez que foi suposto poder produzir apenas um tipo de liga por período.

O dado adicional à primeira modificação é o seguinte:

$\lambda \quad$ qualquer número grande.

A variável adicional é:

$Y_{j t}=\left\{\begin{array}{l}1, \text { se a liga } j \text { for produzida no período } t \\ 0, \text { em caso contrá rio. }\end{array}\right.$

O índice adicional é:

$j=1, \ldots, L \quad$ ligas.

E são considerados os seguintes conjuntos:

$L_{j} \quad$ conjunto das peças produzidas pela liga $j$;

$\bar{L}_{j} \quad$ conjunto das peças não produzidas pela liga $j$.

Foi formulado o problema linear inteiro misto abaixo onde as restrições adicionais (2.23) são restrições lógicas que fazem com que apenas um tipo de liga seja produzida em cada período te (2.21) e (2.22) garantem que somente as peças associadas à liga escolhida possam ser produzidas. 
Minimize $\quad \sum_{t=1}^{T} \sum_{i=1}^{N} \sum_{m=1}^{M} c_{i t} a_{i m} \mathrm{~h}_{t} X_{i m t}$

Sujeito a:

$$
\begin{aligned}
& \sum_{m=1}^{M} a_{i m} h_{t} X_{i m t}-I_{i t}+I_{i(t-1)}=d_{i t} \quad i=1, \ldots, N \quad t=1, \ldots T \\
& \sum_{i=1}^{N} \sum_{m=1}^{M} a_{i m} h_{t} X_{i m t} \leq F_{t} h_{t} \quad t=1, \ldots, T \\
& \sum_{i \in L_{j}} X_{i m t} \leq\left(1-Y_{j t}\right) \lambda+1 \quad j=1, \ldots, L \quad m=1, \ldots, M \quad t=1, \ldots, T \\
& \sum_{i \in L_{j}} X_{i m t} \leq\left(1-Y_{j t}\right) \lambda \quad j=1, \ldots, L \quad m=1, \ldots, M \quad t=1, \ldots, T \\
& \sum_{j=1}^{L} Y_{j t}=1 \quad t=1, \ldots, T \\
& X_{i m t} \geq 0 \quad i=1, \ldots, N \quad m=1, \ldots, M \quad t=1, \ldots, T \\
& Y_{j t} \in\left\{\begin{array}{c}
0,1 \\
j=1, \ldots, L \quad t=1, \ldots, T
\end{array}\right.
\end{aligned}
$$

Este problema pode ser representado, para um período $t$, como visto na figura 2.2, porém com a decisão de que liga utilizar na programação do forno.

\subsubsection{Método de solução}

A matriz de restrições do modelo (2.18)-(2.25) tem uma estrutura semelhante à matriz do primeiro modelo a menos da inclusão submatrizes $Z_{i}$ (coeficientes das variáveis inteiras), conforme mostra a figura abaixo: 


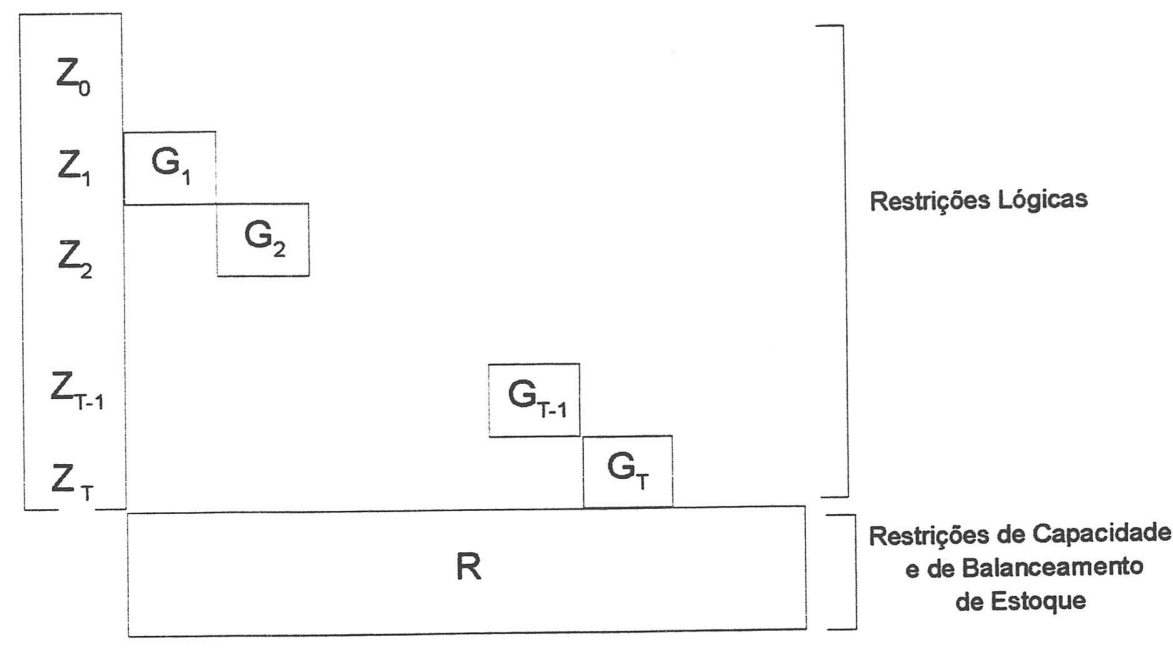

Figura 2.5 Estrutura da matriz de restrições do problema da fundição com várias ligas

Observando a estrutura da matriz de restrições, é sugerido um método heurístico para resolver este problema linear inteiro misto:

Redefinindo $L_{j}=\{1,2, \ldots \ldots, N\}, j=1,2, \ldots L$, (todas as ligas podem produzir todas as peças), que é a heurística proposta por Vianna e Arenales [42], as restrições (2.21), (2.22), bem como as variáveis $Y_{j t}$, tornam-se desnecessárias, já que apenas um tipo de liga é considerado. Desta forma, obtém-se o seguinte programa linear:

Minimize $\sum_{t=1}^{T} \sum_{i=1}^{N} \sum_{m=1}^{M} c_{i t} a_{i m} h_{t} X_{i m t}$

Sujeito a:

$$
\begin{aligned}
& \sum_{m=1}^{M} a_{i m} h_{t} X_{i m t}-l_{i t}+l_{i(t-1)}=d_{i t} \quad i=1, \ldots, N \quad t=1, \ldots T \\
& \sum_{i=1}^{N} \sum_{m=1}^{M} a_{i m} h_{t} X_{i m t} \leq F_{t} h_{t} \quad t=1, \ldots, T \\
& \sum_{i=1}^{N} X_{i m t} \leq 1 \quad m=1, \ldots, M, \quad t=1, \ldots, T \\
& X_{i m t} \geq 0 \quad i=1, \ldots, N, \quad m=1, \ldots, M, \quad t=1, \ldots, T
\end{aligned}
$$


Pode-se observar que este modelo é igual ao problema linear (2.1)-(2.5) proposto para o problema da fundição com uma liga.

Portanto para resolver o problema acima, usa-se o mesmo método de solução proposto na seção 2.2.1. Ou seja, relaxa-se a restrição $\sum_{i=1}^{N} X_{i m t} \leq 1 \mathrm{e}$ define-se a variável $P_{i t}$ como a quantidade de peça $i$ produzida no período $t$, obtendo-se, desta forma, o problema de dimensionamento de lotes monoestágio com limitações de capacidade (2.6)-(2.9), que é um programa linear.

Como foi suposto produzir apenas um tipo de liga por período, deve-se determinar a liga que será produzida em cada período do horizonte de planejamento.

Para determinar tal liga, é necessário verificar se é possível escolher uma liga que permita a produção do conjunto de peças dado pela solução ótima do problema de dimensionamento de lotes monoestágio para o período $t$, ou seja as peças tais que $P_{i t}>0$. Assim, procura-se uma liga $j$ tal que $\left\{i: P_{i t}>0, i=1,2, \ldots, N\right\} \subseteq L_{j}$. Caso não exista uma liga que produza todas as peças dada pela solução ótima do problema (2.6)-(2.9) para o período $t$ adota-se uma heurística. Foram testadas várias heurísticas, entre as que tiveram melhor desempenho destacam-se:

\section{Heurística 1}

Para cada período $t$ :

1. Determine o conjunto das peças que já foram produzidas nos $t$ - 1 períodos anteriores, isto é, calcule:

$$
L_{j_{1}} \cup L_{j_{2}} \cup \ldots \ldots \cup L_{j_{t}-1}
$$

onde $j_{k}$ é a liga $j$ escolhida para o período $k$.

2. Verifique se existe alguma peça que ainda não foi produzida e tem demanda no perícdo $t$, ou seja, verifique para todo i se:

$$
i \notin L_{j_{1}} \cup L_{j_{2}} \cup \ldots \ldots \cup L_{j_{t-1}} \text { e } d_{i t}>0
$$


3. Se existe uma ou mais peças, escolha uma liga que as produza, suponhamos $L_{j_{t}}$. Se houver mais do que uma liga que produza tais peças, faça:

$$
C L_{t}=\left\{j: i \in L_{j} \text { onde } i \text { é det erminado no passo } 2\right\}
$$

E deste conjunto, escolha a liga que produza a maior quantidade de peças dado pela solução ótima do problema de dimensionamento de lotes monoestágio com limitações de capacidade para o período t, isto é:

$$
\max _{j \in C L_{t}}\left\{\sum_{i \in L_{j}} P_{i t}\right\}
$$

Se $C L_{t} \neq 0$, então a heurística não encontrou uma solução.

4. Caso contrário, isto é se todas as peças já foram produzidas nos $t-1$ períodos anteriores, escolha a liga que produza a maior quantidade peças dada pela solução ótima do problema de dimensionamento de lotes monoestágio:

$$
\max _{j=1, \ldots, L}\left\{\sum_{i \in L_{j}} P_{i t}\right\}
$$

\section{Heurística 2}

Para cada período $t$ :

Os passos 1 e 2 são os mesmos da heurística 1

No passo 3, a liga escolhida é aquela que produz o maior número de peças dado pela solução ótima do problema de dimensionamento de lotes monoestágio com limitações de capacidade para o período t, isto é:

$$
\max _{j \in C L_{t}}\left\{\left|\left\{i: P_{i t}>0, i=1, \ldots, N\right\} \cap L_{j}\right|\right\}
$$

onde $|\Omega|$ significa a cardinalidade do conjunto $\Omega$.

E no passo 4, a liga escolhida também é aquela que produz o maior número de peças dada pela solução ótima do problema de dimensionamento de lotes monoestágio, que é a heurística adotada por Vianna e Arenales [42], definida no passo 3 acima. 


\section{Heurística 3}

A heurística 3 é uma combinação das heurísticas 1 e 2. Primeiro é escolhida a liga que produz o maior número de peças dado pela solução do problema monoestágio. Depois é verificado se há empate de ligas, isto é, se há mais de uma liga cujo conjunto de peças têm a mesma cardinalidade, se existir, é definido um novo conjunto com essas ligas e então desse conjunto é escolhida a liga que produz a maior quantidade de peças dada pela solução do problema relaxado.

Se a liga escolhida, por qualquer uma das heurísticas acima, inviabilizou a solução ótima do problema de dimensionamento de lotes monoestágio com limitações de capacidade (problema relaxado), ou seja, haviam peças tais que $P_{i t}>0$ e foram eliminadas, deve-se fixar a liga para o período e resolver o problema de dimensionamento de lotes novamente para que as peças que foram eliminadas sejam produzidas em outro período. Se a liga escolhida permitir a produção do conjunto de peças dado pela solução ótima do problema relaxado para o período $t$ ela é fixada para o período e então é escolhida a liga que será utilizada no próximo período, sem necessidade de resolver novamente o problema com a liga $L_{j_{t}}$ fixada.

Para determinar as ligas dos demais períodos, deve-se repetir o mesmo processo com o problema redefinido, isto é, as ligas dos períodos anteriores devem ter sido escolhidas e fixadas.

Uma vez determinadas as ligas que serão produzidas em cada período, deve-se programar as máquinas, para cada período $t$ do horizonte de planejamento, obtendo desta forma a fração de tempo de produção de cada peça em cada máquina.

O problema de programação das máquinas para cada período $t$ é definido como um problema de transporte generalizado da seguinte forma: 
Minimize $\sum_{m=1}^{M} \sum_{i=1}^{N} X_{i m t}$

Sujeito a

$$
\begin{aligned}
& \sum_{i=1}^{N} X_{i m t} \leq 1 \quad m=1, \ldots, M \\
& \sum_{m=1}^{M} a_{i m} h_{t} X_{i m t} \geq P_{i t} \quad i=1, \ldots, N \\
& \mathrm{X}_{i m t} \geq 0 \quad m=1, \ldots, M \quad i \in L_{j_{\mathrm{t}}} \\
& \mathrm{X}_{\mathrm{imt}}=0 \quad m=1, \ldots, M \quad i \notin L_{j_{\mathrm{t}}}
\end{aligned}
$$

onde $j_{t}$ é a liga escolhida para o período $t$.

Este problema, para um período t onde liga escolhida não produz a peça 2, pode ser representado pela figura abaixo:

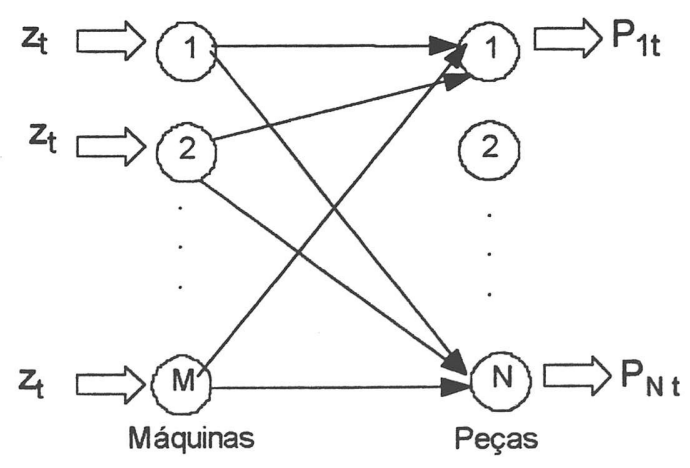

Figura 2.60 problema de transporte generalizado para a - programação das máquinas

Aqui, como no problema da fundição com uma liga, pode-se usar o modelo da máxima utilização das máquinas. Desta forma o modelo para a programação das máquinas para o período $t$ é: 
Minimize $z_{t}$

Sujeito a

$$
\begin{aligned}
& \sum_{i=1}^{N} X_{i m t} \leq z_{t} \quad m=1, \ldots, M \\
& \sum_{m=1}^{M} a_{i m} h_{t} X_{i m t} \geq P_{i t} \quad i=1, \ldots, N \\
& X_{i m t} \geq 0 \quad m=1, \ldots, M i \in L_{j_{t}} \\
& X_{i m t}=0 \quad m=1, \ldots, M i \notin L_{j_{t}}
\end{aligned}
$$

Da mesma forma anterior, observe que este problema é factivel se a variável $z_{t} \leq 1$

\subsubsection{Algoritmo}

- Passo 0

Faça $t=1$.

\section{- Passo 1}

Resolver o problema de dimensionamento de lotes monoestágio com limitações de capacidade, (2.6)-(2.9).

\section{- Passo 2}

Determinar a liga $j_{t}$ que será utilizada no período $t$ usando uma das heurísticas descritas na seção 2.3.1

\section{- Passo 3}

Fixar $P_{i t}=0, i \notin L_{j_{t}}$

Fazer $t=t+1$. 
Se $t>T$ então ir para o passo 4.

Se $\left\{i \mid P_{i t}>0, i=1, \ldots, N\right\} \subseteq L_{j_{\mathrm{t}}}$ ir para passo 2 , caso contrário ir para o passo 1.

- Passo 4

Resolver o problema de dimensionamento de lotes monoestágio com limitações de capacidade.(Lembre-se que as ligas $j_{1}, j_{2}, \ldots ., j_{T}$ já foram escolhidas para os períodos $1,2, \ldots ., T$, respectivamente).

Para cada período $t, t=1, \ldots, T$, resolver o problema (2.26)-(2.30) ou 0 problema (2.31)-(2.35), determinando $X_{i m t}$, a fração de tempo utilizada para produzir peças do tipo i na máquina $m$. Fim.

\subsubsection{Experiências computacionais}

Para a resolução do algoritmo apresentado na seção 2.3.2, as três heurísticas foram implementadas em linguagem C. Para a resolução do problema de dimensionamento de lotes monoestágio com limitações de capacidade e os problemas (2.26)-(2.30) e por (2.31)-(2.35) foi implementado um programa em $C$ que utiliza rotinas do pacote CPLEX. Foram utilizadas as seguintes rotinas deste pacote: para a alocação dos dados foi utilizada a rotina loadlp, para encontrar a solução ótima destes problemas foi utilizada a rotina optimize (que otimiza um problema linear usando o primal simplex), para acessar os valores ótimos da solução dos problemas foi utilizada a rotina solution e, para fazer $P_{i t}=0$ depois de se ter determinado a liga para o periodo $t$ foi utilizada a rotina chgbds que muda os limitantes das variáveis. O compilador utilizado foi o gcc para estações de trabalho.

Também foi feito um programa em $\mathrm{C}$ que escreve o programa linear inteiro misto na forma lp e compilado no compilador BCPLUS, versão 3.1. A forma lp é uma das maneiras pela qual o CPLEX lê um problema a ser otimizado em que não há necessidades de mudanças posteriores dos dados do problema.

Observe que o número de variáveis contínuas do problema linear inteiro misto (2.18) - (2.25) é $T\left(M^{*} N+N\right)$, o número de variáveis zero-um é $T^{\star} J$ e o número de restrições é $T\left(N+2+2\left(J^{*} M\right)\right)$. 
No apêndice 1 há um exemplo de um problema resolvido inteiro e também pelo método heurístico proposto, com a programação das máquinas feita por (2.26)-(2.30) e por (2.31)-(2.35).

Neste caso, para problemas pequenos o pacote CPLEX foi mais eficiente do que a heurística, conseguindo aproximadamente uma solução $0.5 \%$ melhor do que a solução heurística, em 8 exemplos com pequenas variações no problema apresentado no apêndice 1. Com estes testes pode-se observar a heurística é confiável.

Para problemas maiores, por exemplo, onde $N=25, T=8, J=8$ e $M=10,0$ CPLEX não conseguiu uma solução factível para o problema linear inteiro misto em cinco horas de execução. O método heurístico proposto conseguiu uma solução factivel em 30 segundos. Deve-se observar que o CPLEX usa uma heurística, que fixa inicialmente algumas variáveis inteiras cujos valores obtidos pela resolução do problema relaxado estão próximos de valores inteiros, com isto o problema tornouse infactivel.

Através destes exemplos, pode-se observar a importância de se ter método heurísticos baseados em problemas. 


\section{CAPÍTULO 3}

\section{Um problema de dimensionamento de lotes com múltiplos estágios e com limitações de capacidade}

\subsection{Introdução}

Este capítulo revisa a formulação matemática do problema de dimensionamento de lotes com múltiplos estágios e com limitações de capacidade, proposto por Santos [3], que a princípio, foi modelado para os estágios da usinagem e montagem de uma indústria de autopeças do interior paulista, mas que pode representar, além desses, outros estágios. Ainda neste capítulo, é feita a "integração" deste programa com o problema da fundição, que neste caso é visto como mais um estágio de tal indústria. 


\subsection{Formulação matemática do problema de dimensionamento de lotes com múltiplos estágios e com limitações de capacidade}

O problema consiste em produzir vários tipos de itens, os quais passam pelos vários estágios de produção, os quais têm estruturas similares de operação. Em cada período pode-se produzir diferentes itens finais e intermediários. Os itens finais são destinados à venda externa (demanda externa), enquanto que, alguns itens intermediários são utilizados tanto para compor um novo item (demanda interna) quanto para a venda externa (demanda externa). Para produzir itens finais e/ou itens intermediários, em cada estágio, pode ser necessário, além de itens produzidos nos estágios anteriores, insumos externos. É suposto que estes insumos (matérias primas do tipo: parafusos, roldanas, etc.) possam ser conseguidos sem restrições de mercado sobre eles, de modo que não se fará necessário um planejamento de aquisição.

O objetivo do modelo é determinar o quanto deve ser produzido de cada item em cada período, submetendo-se as restrições de capacidade de produção da fábrica, tendo em vista a capacidade de absorção do mercado, de modo a maximizar o lucro líquido. O planejamento deve ser feito em vários períodos, para os quais são conhecidos intervalos de demanda. Não foram considerados custos e tempos de preparações (setup). As capacidades das máquinas (centro de trabalho) são variáveis de decisão do problema, que dependem do número de pessoas alocadas.

Pode-se representar o problema através da figura 3.1. Foram considerados dois estágios de produção: usinagem e montagem.

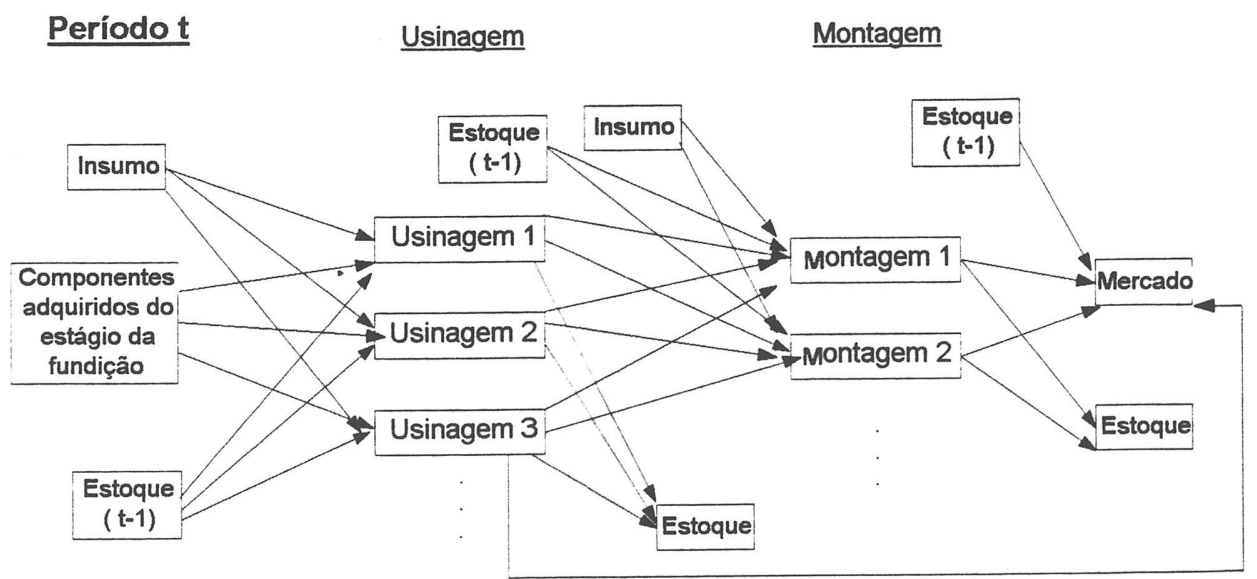

Figura 3.1 Representação esquemática do processo de produção. 
Pode-se observar que a estrutura geral de produto é intrínseca ao problema, onde os insumos, os componentes adquiridos do estágio anterior e o estoque $(t-1)$ correspondem às matérias primas, a usinagem e a montagem correspondem aos estágios de produção.

Considerando esta figura pode-se notar que algumas matérias-primas do estágio da usinagem são na realidade o produto final do estágio da fundição (juntamente com outras matérias-primas que não são fabricadas pela fundição existente na indústria (insumos)). Pode-se observar que a fundição não está incorporada neste modelo porque tem uma estrutura de funcionamento diferente.

Para a formulação do modelo foi considerada a seguinte notação.

Dados:

$\underline{d}_{\text {it }}$ demanda externa mínima do item i no período $t$.

$\bar{d}_{i t} \quad$ demanda externa máxima do item i no período $t$.

$\alpha_{i t}$ preço de venda de uma unidade do item $i$ no período $t$.

$\beta_{i t} \quad$ custo de produção de uma unidade do item $i$ no período $t$.

$\delta_{i t} \quad$ custo de estoque de uma unidade do item $i$ no período $t$.

$b_{i k} \quad$ tempo necessário para produzir uma unidade do item i no centro de trabalho $k$.

$a_{i j} \quad$ quantidade do item $i$ necessária para produzir uma unidade do item $j$.

$\omega_{t k}$ custo de uma unidade de capacidade alocada no periodo $t$ no centro de trabalho $k$.

$h_{t}$ quantidade total de capacidade disponível (em unidades de tempo) no período $t$.

$M O_{t k}$ quantidade máxima de capacidade alocável (em unidades de tempo) no centro de trabalho $k$ no período $t$. 
$g_{1 j}$ quantidade necessária de insumo / para produzir uma unidade do item $j$.

Variáveis de decisão:

$X_{i t} \quad$ quantidade do item $i$ produzida no período $t$.

$I_{i t} \quad$ quantidade do item $i$ estocada no final do período $t$.

$V_{i t} \quad$ quantidade do item $i$ vendida no período $t$.

$X_{i t k}$ quantidade do item $i$ produzida no período $t$ no centro de trabalho $k$.

$H_{t k} \quad$ capacidade disponível no período $t$ no centro de trabalho $k$.

$Q_{1 t} \quad$ quantidade de insumo / estocada no final do período $t$.

$\Psi_{l t} \quad$ quantidade do insumo / comprada no período $t$.

Índices:

$i=1, \ldots, N \quad$ itens

$k=1, \ldots, k \quad$ centros de trabalho.

$t=1, \ldots ., T$ períodos.

$I=1, \ldots, L \quad$ insumos

Conjuntos de índices:

$P_{i} \quad$ conjunto dos predécessores imediatos do item $i$.

$S_{i} \quad$ conjunto dos sucessores imediatos do item $i$.

$K_{i} \quad$ conjunto dos centros de trabalho que podem produzir o item $i$. 
Pelas experiências práticas da fábrica, foi observado que a capacidade dos centros de trabalho, em cada período, depende do número de pessoas designadas para estes. Seja $m_{k}$ a capacidade (em unidades de tempo) do centro de trabalho $k$ para cada pessoa alocada. A capacidade total, para cada centro de trabalho foi aproximada linearmente por $H_{t k}=H_{t k}\left(N_{t k}\right)=m_{k} N_{t k}$, onde $N_{t k}$ é o número de pessoas utilizadas no centro de trabalho $k$ no período $t$. Foi observado que o aumento do número de pessoas alocadas num centro de trabalho tende a saturarse de modo a decair a produtividade. Para ilustrar o rendimento de capacidade no centro de trabalho e o número de pessoas designadas, foi construído o gráfico dado pela figura 3.2 .

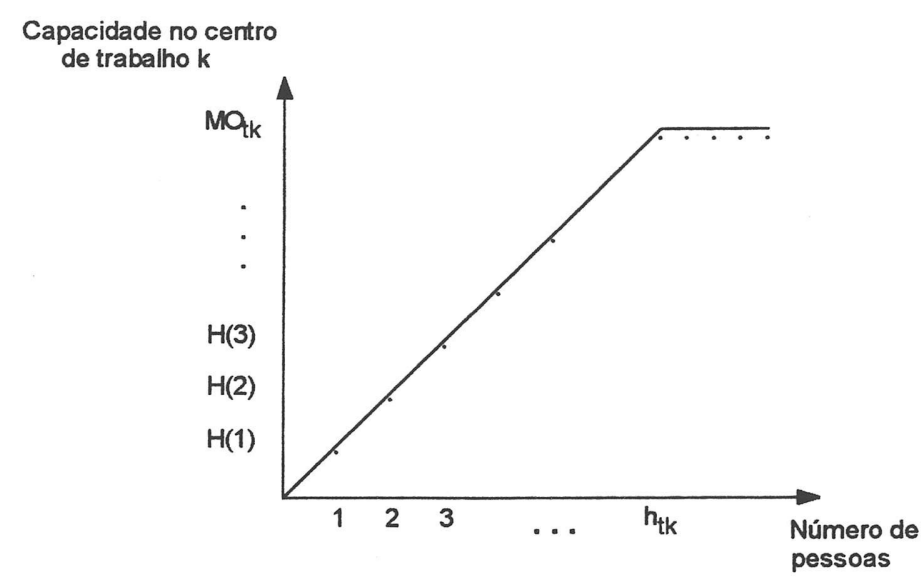

Figura 3.2 Representação da relação entre a capacidade do centro de trabalho e número de pessoas alocadas

O ponto de saturação é suposto conhecido e modelado pela restrição $N_{t k} \leq \eta_{t k}$ para cada centro de trabalho $k$ e cada período $t$, onde $\eta_{t k}$ é o número máximo possível de pessoas alocáveis no centro de trabalho $k$ no período $t$. De onde segue que $M O_{t k}=m_{k} \eta_{t k}$ é a capacidade máxima alocável ao centro de trabalho $k$ no periodo $t$.

Foi proposto pelos autores o seguinte problema linear: 
Maximize $Z=\sum_{t=1}^{T}\left[\sum_{i=1}^{N}\left(\alpha_{i t} V_{i t}-\beta_{i t} X_{i t}-\delta_{i t} J_{i t}\right)-\sum_{k=1}^{K} \omega_{t k} H_{t k}\right]$

Sujeito a:

$V_{i t}+I_{i t}+\sum_{j \in S_{i}} a_{1 j} X_{j t}=X_{i t}+I_{i(t-1)} \quad i=1, \ldots, N \quad t=1, \ldots, T$

$X_{i t}=\sum_{k \in K_{i}} X_{i t k} \quad i=1, \ldots, N \quad t=1, \ldots, T$

$\sum_{i=1}^{N} b_{i k} X_{i t k} \leq H_{t k} \quad k=1, \ldots, K \quad t=1, \ldots, T$

$Q_{t l}+\sum_{j=1}^{N} g_{l j} X_{j t}=\Psi_{t t}+Q_{(t-1) !} \quad l=1, \ldots, L \quad t=1, \ldots, T$

$\sum_{k=1}^{K} H_{t k} \leq h_{t} \quad t=1, \ldots, T$

$H_{t k} \leq M O_{t k} \quad k=1, \ldots, K \quad t=1, \ldots, T$

$\underline{d}_{i t} \leq V_{i t} \leq \bar{d}_{i t} i=1, \ldots, N \quad t=1, \ldots, T$

$X_{i t}, I_{i t}, X_{i t k}, H_{t k}, Q_{t l}, \Psi_{t l} \geq 0 \quad i=1, . ., N \quad t=1, . ., \quad k=1, . ., K \quad I=1, . . L$

$I_{i 0}=0 \quad$ e $\quad I_{i T}=0 \quad i=1, \ldots, N$

A função objetivo (3.1) representa o lucro líquido que deve ser maximizado; as equações (3.2) são as restrições de balanceamento de estoque da produção de cada item no final de cada período do horizonte de planejamento, as equações (3.3) definem que a produção total de cada item em cada período; as inequações (3.4) são as restrições de capacidade da fábrica; as inequações; (3.5) são as 
restrições de balanceamento de estoque dos insumos, (como não existem limitações de mercado sobre os insumos, estas restrições de balanceamento de estoque dos insumo, (3.5), foram relaxadas (abandonadas), sem alterar a solução do problema. Depois do problema ser resolvido, pode-se facilmente calcular o balanceamento de estoque dos mesmos); (3.6) são as restrições de limitação de capacidade em cada período, (3.7) são as restrições de capacidade máxima alocável em cada centro de trabalho, (3.8) são as restrições de mercado, (3.9) são as restrições de não-negatividade e (3.10) são as condições de contorno do problema.

Este modelo difere do modelo apresentado em Billington et al.[9] nos seguintes aspectos:

- a demanda é restrita a um intervalo (são especificados valores máximo e mínimo);

- a capacidade de cada centro de trabalho é uma variável de decisão;

- o objetivo é maximizar o lucro líquido;

- são consideradas as variáveis $X_{i t k}$ que definem a produção por máquina;

- não são considerados o tempo e o custo de preparação.

\subsection{Integração do estágio da fundição com os estágios da usinagem e montagem}

O problema integrado pode ser representado como visto na figura 3.3 , onde o problema da fundição pode ser considerado o problema com uma, ou várias, ligas, para um determinado periodo t:

Em ambos os casos pode-se observar que a demanda do estágio da fundição é parte da matéria-prima do estágio da usinagem, portanto quando se resolve o problema da fundição, a demanda da fundição é o requerimento do estágio da usinagem e, anteriormente, no capítulo 2 , a demanda era considerada como um dado do problema. 
Períodot

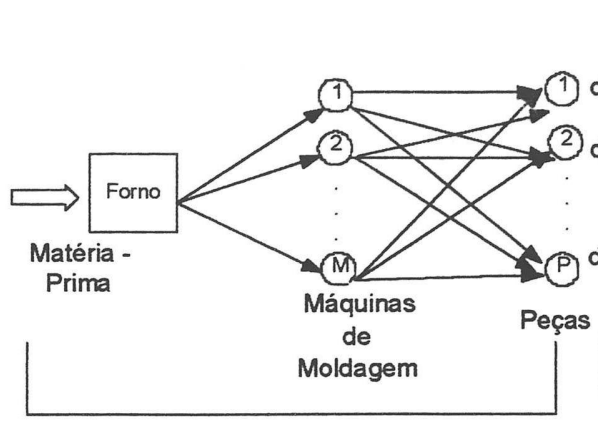

Fundição

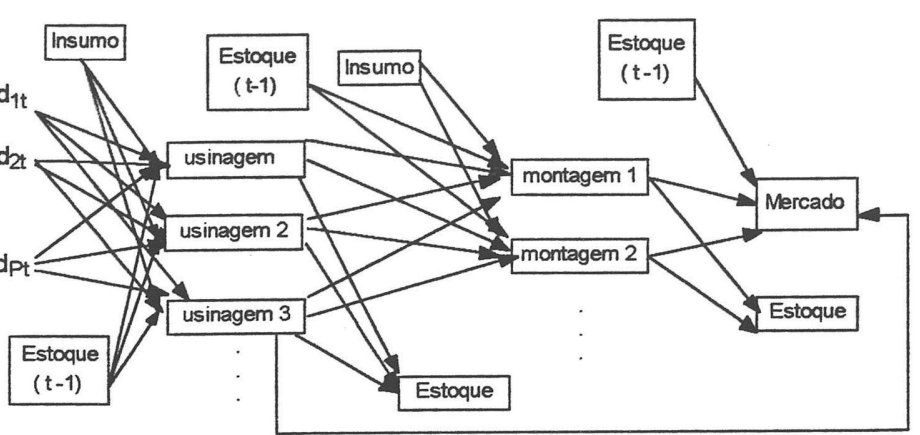

Usinagem
Montagem

Figura 3.30 problema integrado

\subsection{Método de solução}

As observações da seção anterior sugerem um método de solução para este problema, onde deve-se primeiramente resolver $\circ$ problema de dimensionamento de lotes, modelo (3.1)-(3.10) e depois resolver um dos problemas da fundição.

\section{Algoritmo}

\section{- Passo 1}

Resolva o problema de dimensionamento de lotes com múltiplos estágios e com limitações de capacidade, modelo (3.1)-(3.10), obtendo-se $X_{i t}, i=1, . ., N$ e $\mathrm{t}=$ $1, \ldots, N$.

\section{- Passo 2}

Sejam $\{N-u, N-u+1, \ldots ., N\}$ os itens que devem ser produzidos pela fundição.

$$
\text { Faça } d_{i t}=X_{i t}, i=N-u, N-u+1, \ldots, N
$$

\section{- Passo 3}

Resolva um dos problemas da fundição por um dos métodos de solução apresentados nas seções 2.2.1 ou 2.3.1 do capítulo 2, determinando a produção 
das peças que serão, juntamente com outros insumos, a matéria-prima do estágio da usinagem.

Se o gargalo da indústria estiver nos estágios da usinagem ou montagem (ou seja, se a capacidade de produção da fundição é folgada em relação a capacidade de produção da usinagem e montagem) este procedimento deverá produzir bons resultados ('a semelhança do método para resolver o problema da fundição). Caso contrário, o problema da fundição pode ser inviável. 


\section{COMENTÁRIOS FINAIS E CONCLUSÕES}

Neste trabalho foram estudados problemas de dimensionamento de lotes com aplicações específicas numa fundição automatizada.

Dois casos foram estudados, com uma liga e com múltiplas ligas, para os quais foram apresentados modelos matemáticos e métodos heurísticos de resolução baseados nas estruturas dos problemas.

No caso de uma liga, o modelo é linear com a matriz de restrições esparsa e sob a hipótese do forno ser o gargalo de produção a heurística sempre produz soluções ótimas. No caso de várias ligas o modelo é um linear inteiro misto para o qual é proposto um método heurístico.

A heurística para a escolha da liga (Heurística 1), seção 2.3.1, apresentou melhores resultados do que as demais heurísticas apresentadas na mesma seção. Pode-se observar a qualidade da solução da heurística e concluir que ela está aproximadamente $0.5 \%$ do valor da solução ótima inteira mista encontrada pelo CPLEX, em exemplos pequenos.

Para problemas maiores (por exemplo, com 2200 variáveis contínuas, 160 variáveis 0-1 e 856 restrições), o CPLEX não obteve solução factível após cinco horas de execução (devido ao fato que a heurística que o mesmo usa para fixar algumas variáveis inteiras faliiou), enquanto que a heurística 1 obteve uma solução factível (verificada explicitamente) em aproximadamente trinta segundos.

A fundição pode ser vista como um estágio a mais num sistema de manufatura com múltiplos estágios e os estudos realizados neste trabalho, capítulo 
2, podem ser integrados num modelo geral de dimensionamento de lotes com múltiplos estágios conforme visto no capítulo 3. Este é um tópico para pesquisas futuras, devendo-se desenvolver métodos onde a solução heurística obtida para a fundição e a solução para os estágios subsequentes sejam refinadas. 


\section{APENDICE 1}

\section{Exemplo de um problema da fundição com várias ligas}

\section{Dados:}

Número de máquinas: $m=1, \ldots, 5$

Número de peças: $i=1, \ldots, 10$

Número de períodos: $t=1, \ldots, 4$

Número de ligas $j=1, \ldots, 5$

Quantidade de peças i possíveis de serem produzidas na máquina m no período $t_{,}\left(a_{i}\right.$ m), (toneladas/hora):

$\begin{array}{lllll}a_{11}=8.0 & a_{12}=8.0 & a_{13}=12.0 & a_{14}=16.0 & a_{15}=20.0 \\ a_{21}=8.0 & a_{22}=12.0 & a_{23}=16.0 & a_{24}=20.0 & a_{25}=12.0 \\ a_{31}=12.0 & a_{32}=16.0 & a_{33}=20.0 & a_{34}=8.0 & a_{35}=16.0 \\ a_{41}=16.0 & a_{42}=20.0 & a_{43}=12.0 & a_{44}=8.0 & a_{45}=8.0 \\ a_{51}=20.0 & a_{52}=8.0 & a_{53}=12.0 & a_{54}=16.0 & a_{65}=8.0 \\ a_{61}=8.0 & a_{62}=12.0 & a_{63}=16.0 & a_{64}=20.0 & a_{65}=12.0 \\ a_{71}=12.0 & a_{72}=16.0 & a_{73}=20.0 & a_{74}=8.0 & a_{75}=12.0 \\ a_{81}=16.0 & a_{82}=20.0 & a_{83}=12.0 & a_{84}=8.0 & a_{85}=8.0 \\ a_{91}=20.0 & a_{92}=12.0 & a_{93}=16.0 & a_{94}=8.0 & a_{95}=12.0 \\ a_{101}=16.0 & a_{102}=20.0 & a_{103}=8.0 & a_{104}=12.0 & a_{105}=20.0\end{array}$

Conjunto das peças produzidas pela liga $\mathrm{j}$ :

$$
\begin{array}{ll}
L_{1}=\{2,4,5,8,9,10\}, & L_{2}=\{1,3,6,7\}, \quad L_{3}=\{1,2,3,4,6,8,9\} \\
L_{4}=\{1,3,5,7,8,10\}, & L_{5}=\{2,4,5,6,7,9,10\}
\end{array}
$$

Conjunto das peças não produzidas pela liga j:

$$
\begin{array}{ll}
L_{1}^{\prime}=\{1,3,6,7\}, & L_{2}^{\prime}=\{2,4,5,8,9,10\}, \quad L_{3}^{\prime}=\{5,7,10\} \\
L^{\prime}=\{2,4,6,9\}, & L_{5}^{\prime}=\{1,3,8\}
\end{array}
$$


Número de horas de cada periodo, $\left(h_{t}\right)$, (horas):

$$
h_{1}=12 \quad h_{2}=10 \quad h_{3}=10 \quad h_{4}=12
$$

Capacidade do forno $\left(F_{t}\right)$, (toneladas/hora)

$$
F_{1}=80 \quad F_{2}=80 \quad F_{3}=80 \quad F_{4}=80
$$

Demanda da peça i no período $t,\left(d_{i t}\right)$, (toneladas/período):

$\begin{array}{llll}d_{11}=0.0 & d_{12}=160.0 & d_{13}=200.0 & d_{14}=100.0 \\ d_{21}=0.0 & d_{22}=240.0 & d_{23}=120.0 & d_{24}=60.0 \\ d_{31}=0.0 & d_{32}=140.0 & d_{33}=250.0 & d_{34}=80.0 \\ d_{41}=0.0 & d_{42}=100.0 & d_{43}=100.0 & d_{44}=50.0 \\ d_{51}=0.0 & d_{52}=0.0 & d_{53}=100.0 & d_{54}=30.0 \\ d_{61}=0.0 & d_{62}=0.0 & d_{63}=10.0 & d_{64}=150.0 \\ d_{71}=0.0 & d_{72}=200.0 & d_{73}=20.0 & d_{74}=0.0 \\ d_{81}=0.0 & d_{82}=0.0 & d_{83}=30.0 & d_{84}=100.0 \\ d_{91}=0.0 & d_{92}=0.0 & d_{93}=140.0 & d_{94}=20.0 \\ d_{101}=0.0 & d_{102}=300.0 & d_{103}=10.0 & d_{104}=50.0\end{array}$

Custo de produção da peça i no período $t\left(c_{i t}\right),(\$)$ :

$\begin{array}{llll}c_{11}=180.0 & c_{12}=160.0 & c_{13}=140.0 & c_{14}=100.0 \\ c_{21}=180.0 & c_{22}=160.0 & c_{23}=140.0 & c_{24}=100.0 \\ c_{31}=180.0 & c_{32}=160.0 & c_{33}=140.0 & c_{34}=100.0 \\ c_{41}=180.0 & c_{42}=160.0 & c_{43}=140.0 & c_{44}=100.0 \\ c_{51}=180.0 & c_{52}=160.0 & c_{53}=140.0 & c_{54}=100.0 \\ c_{61}=180.0 & c_{62}=160.0 & c_{63}=140.0 & c_{64}=100.0 \\ c_{71}=180.0 & c_{72}=160.0 & c_{73}=140.0 & c_{74}=100.0 \\ c_{81}=180.0 & c_{82}=160.0 & c_{83}=140.0 & c_{84}=100.0 \\ c_{91}=180.0 & c_{92}=160.0 & c_{93}=140.0 & c_{94}=100.0 \\ c_{101}=180.0 & c_{102}=160.0 & c_{103}=140.0 & c_{104}=100.0\end{array}$

\section{Problema linear inteiro misto ((2.18) - (2.25))}

Pode-se observar que o número de variáveis deste problema é 260 e o número de restrições é 248. Portanto é um problema bem pequeno.

O CPLEX encontrou a solução ótima para este problema linear inteiro misto. Esta solução está relacionada abaixo: 
Valor Objetivo: $\mathbf{4 0 4 . 0 0 0}$

\section{Período 1}

Produção de peças no período 1 utilizando a liga 5

\begin{tabular}{|c|c|c|c|c|}
\hline & Peça & Máquina & Tempo & Quant/Maq \\
\hline & 2 & 2 & 0.347222 & 50 \\
\hline & 2 & 4 & 1.0 & 240 \\
\hline & 4 & 2 & 0.416667 & 100 \\
\hline & 10 & 1 & 1.0 & 192 \\
\hline & 10 & 2 & 0.075 & 18 \\
\hline Total & & & 2.83889 & 600 \\
\hline
\end{tabular}

\begin{tabular}{|c|c|c|c|}
\hline Peça & $\begin{array}{c}\text { Quantidade } \\
\text { produzida }\end{array}$ & Demanda & Estoque $\mathrm{t}=1$ \\
\hline 1 & 0 & 0 & 0 \\
\hline 2 & 290 & 0 & 290 \\
\hline 3 & 0 & 0 & 0 \\
\hline 4 & 100 & 0 & 100 \\
\hline 5 & 0 & 0 & 0 \\
\hline 6 & 0 & 0 & 0 \\
\hline 7 & 0 & 0 & 0 \\
\hline 8 & 0 & 0 & 0 \\
\hline 9 & 0 & 0 & 0 \\
\hline 10 & 210 & 0 & 210 \\
\hline
\end{tabular}

\section{Período 2}

Produção de peças no período 2 utilizando a liga 4

\begin{tabular}{|c|c|c|c|c|}
\hline & Peça & Máquina & Tempo & Quant/Maq \\
\hline & 1 & 1 & 0.466667 & 37.3336 \\
\hline & 1 & 3 & 0.3 & 36 \\
\hline & 1 & 4 & 0.229167 & 36.66672 \\
\hline & 1 & 5 & 0.25 & 50 \\
\hline & 3 & 3 & 0.70 & 140 \\
\hline & 5 & 1 & 0.03333 & 6.66 \\
\hline & 5 & 4 & 0.770833 & 123.34 \\
\hline & 7 & 1 & 0.50 & 60 \\
\hline & 7 & 2 & 1.0 & 160 \\
\hline & 10 & 5 & 0.75 & 150 \\
\hline & & & 4.999967 & 800.0 \\
\hline
\end{tabular}




\begin{tabular}{|c|c|c|c|c|}
\hline Peça & $\begin{array}{c}\text { Quantidade } \\
\text { produzida }\end{array}$ & Estoque $\mathrm{t}=1$ & Demanda & Estoque $\mathrm{t}=\mathbf{2}$ \\
\hline 1 & 160 & 0 & 160 & 0 \\
\hline 2 & 0 & 290 & 240 & 50 \\
\hline 3 & 140 & 0 & 140 & 0 \\
\hline 4 & 0 & 100 & 100 & 0 \\
\hline 5 & 130 & 0 & 0 & 130 \\
\hline 6 & 0 & 0 & 0 & 0 \\
\hline 7 & 220 & 0 & 200 & 20 \\
\hline 8 & 0 & 0 & 0 & 0 \\
\hline 9 & 0 & 0 & 0 & 60 \\
\hline 10 & 150 & 210 & 300 & \\
\hline
\end{tabular}

\section{Período 3}

Produção de peças no período 3 utilizando a liga 3

\begin{tabular}{|c|c|c|c|c|}
\hline & Peça & Máquina & Tempo & Quant/Máq \\
\hline & 1 & 4 & 0.95 & 152 \\
\hline & 1 & 5 & 0.24 & 48 \\
\hline & 2 & 1 & 0.015 & 1.2 \\
\hline & 2 & 3 & 0.43 & 68.8 \\
\hline & 3 & 2 & 0.85 & 136 \\
\hline & 3 & 3 & 0.57 & 114 \\
\hline & 4 & 1 & 0.625 & 100 \\
\hline & 6 & 4 & 0.050 & 10 \\
\hline & 8 & 2 & 0.15 & 30 \\
\hline & 9 & 1 & 0.244 & 48.8 \\
\hline & 9 & 5 & 0.76 & 91.2 \\
\hline & & & 4.884 & 800 \\
\hline
\end{tabular}

\begin{tabular}{|c|c|c|c|c|}
\hline Peça & $\begin{array}{c}\text { Quantidade } \\
\text { produzida }\end{array}$ & Estoque $\mathrm{t}=2$ & Demanda & Estoque $\mathrm{t}=3$ \\
\hline 1 & 200 & 0 & 200 & 0 \\
\hline 2 & 70 & 50 & 120 & 0 \\
\hline 3 & 250 & 0 & 250 & 0 \\
\hline 4 & 100 & 0 & 100 & 0 \\
\hline 5 & 0 & 130 & 100 & 30 \\
\hline 6 & 10 & 0 & 10 & 0 \\
\hline 7 & 0 & 20 & 20 & 0 \\
\hline 8 & 30 & 0 & 30 & 0 \\
\hline 9 & 140 & 0 & 140 & 50 \\
\hline 10 & 0 & 60 & 10 & 0 \\
\hline
\end{tabular}




\section{Período 4}

Produção de peças no período 4 utilizando a liga 3

\begin{tabular}{|c|c|c|c|c|}
\hline & Peça & Máquina & Tempo & Quant/Máq \\
\hline & 1 & 1 & 0.010417 & 1 \\
\hline & 1 & 3 & 0.6875 & 99 \\
\hline & 2 & 3 & 0.3125 & 60 \\
\hline & 3 & 2 & 0.416667 & 80 \\
\hline & 4 & 4 & 0.520833 & 50 \\
\hline & 6 & 1 & 0.0625 & 6 \\
\hline & 6 & 5 & 1.0 & 144 \\
\hline & 8 & 1 & 0.520833 & 100 \\
\hline & 9 & 1 & 0.08333 & 20 \\
\hline & & & 3.61458 & 560 \\
\hline
\end{tabular}

\begin{tabular}{|c|c|c|c|c|}
\hline Peça & $\begin{array}{c}\text { Quantidade } \\
\text { produzida }\end{array}$ & Estoque t=3 & Demanda & Estoque $\mathrm{t}=4$ \\
\hline 1 & 100 & 0 & 100 & 0 \\
\hline 2 & 60 & 0 & 60 & 0 \\
\hline 3 & 80 & 0 & 80 & 0 \\
\hline 4 & 50 & 0 & 50 & 0 \\
\hline 5 & 0 & 30 & 30 & 0 \\
\hline 6 & 150 & 0 & 150 & 0 \\
\hline 7 & 0 & 0 & 0 & 0 \\
\hline 8 & 100 & 0 & 100 & 0 \\
\hline 9 & 20 & 0 & 20 & 0 \\
\hline 10 & 0 & 50 & 50 & 0 \\
\hline
\end{tabular}

\section{Método de Solução Heurístico Proposto}

Pode-se notar que o número de variáveis do problema da dimensionamento de lotes monoestágio com limitações de capacidade (2.6)-(2.9) é 80 e o número de restrições é 44. E, cada um dos problemas (2.26)-(2.30) e (2.31)-(2.35) têm 50 variáveis e 15 restrições. 
Resolução do problema acima utilizando a Heurística 1 e a programação das máquinas efetuada pelo modelo (2.26)-(2.30).

Valor Objetivo: 406.200

Período 1

Produção de peças no período 1 utilizando a liga 5

\begin{tabular}{|c|c|c|c|c|}
\hline & Peça & Máquina & Tempo & Quant/Maq \\
\hline & 5 & 1 & 0.541667 & 130 \\
\hline & 7 & 3 & 0.91667 & 220 \\
\hline & 10 & 2 & 0.50 & 120 \\
\hline Total & 10 & 5 & 1.0 & 240 \\
\hline
\end{tabular}

\begin{tabular}{|c|c|c|c|}
\hline Peça & $\begin{array}{c}\text { Quantidade } \\
\text { produzida }\end{array}$ & Demanda & Estoque $\mathrm{t}=1$ \\
\hline 1 & 0 & 0 & 0 \\
\hline 2 & 0 & 0 & 0 \\
\hline 3 & 0 & 0 & 0 \\
\hline 4 & 0 & 0 & 0 \\
\hline 5 & 130 & 0 & 130 \\
\hline 6 & 0 & 0 & 0 \\
\hline 7 & 220 & 0 & 220 \\
\hline 8 & 0 & 0 & 0 \\
\hline 9 & 0 & 0 & 0 \\
\hline 10 & 360 & 0 & 360 \\
\hline
\end{tabular}

\section{Período 2}

Produção de peças no período 2 utilizando a liga 3

\begin{tabular}{|c|c|c|c|c|}
\hline & Peça & Máquina & Tempo & Quant/Maq \\
\hline & 1 & 5 & 0.80 & 160 \\
\hline & 2 & 3 & 0.3125 & 50 \\
\hline & 2 & 4 & 0.95 & 190 \\
\hline & 3 & 3 & 0.6875 & 137.5 \\
\hline & 3 & 5 & 0.015625 & 2.5 \\
\hline & 4 & 2 & 0.7 & 140 \\
\hline & 6 & 4 & 0.050 & 10 \\
\hline & & & 3.515625 & 690 \\
\hline
\end{tabular}




\begin{tabular}{|c|c|c|c|c|}
\hline Peça & Quantidade & Estoque $\mathrm{t}=1$ & Demanda & Estoque $\mathrm{t}=\mathbf{2}$ \\
\hline 1 & 160 & 0 & 160 & 0 \\
\hline 2 & 240 & 0 & 240 & 0 \\
\hline 3 & 140 & 0 & 140 & 0 \\
\hline 4 & 140 & 0 & 100 & 40 \\
\hline 5 & 0 & 130 & 0 & 130 \\
\hline 6 & 10 & 0 & 0 & 10 \\
\hline 7 & 0 & 220 & 200 & 20 \\
\hline 8 & 0 & 0 & 0 & 0 \\
\hline 9 & 0 & 0 & 0 & 0 \\
\hline 10 & 0 & 360 & 300 & 60 \\
\hline
\end{tabular}

\section{Período 3}

Produção de peças no período 3 utilizando a liga 3

\begin{tabular}{|c|c|c|c|c|}
\hline & Peça & Máquina & Tempo & Quant/Máq \\
\hline & 1 & 5 & 1.0 & 200 \\
\hline & 2 & 4 & 0.60 & 120 \\
\hline & 3 & 2 & 0.3125 & 50 \\
\hline & 3 & 3 & 1.0 & 200 \\
\hline & 4 & 2 & 0.30 & 60 \\
\hline & 8 & 2 & 0.15 & 30 \\
\hline & 9 & 1 & 0.7 & 140 \\
\hline & & & 4.0625 & 800 \\
\hline
\end{tabular}

\begin{tabular}{|c|c|c|c|c|}
\hline Peça & Quantidade & Estoque $t=2$ & Demanda & Estoque $t=3$ \\
\hline 1 & 200 & 0 & 200 & 0 \\
\hline 2 & 120 & 0 & 120 & 0 \\
\hline 3 & 250 & 0 & 250 & 0 \\
\hline 4 & 60 & 40 & 100 & 0 \\
\hline 5 & 0 & 130 & 100 & 30 \\
\hline 6 & 0 & 10 & 10 & 0 \\
\hline 7 & 0 & 20 & 20 & 0 \\
\hline 8 & 30 & 0 & 30 & 0 \\
\hline 9 & 140 & 0 & 140 & 0 \\
\hline 10 & 0 & 60 & 10 & 50 \\
\hline
\end{tabular}




\section{Período 4}

Produção de peças no período 4 utilizando a liga 3

\begin{tabular}{|c|c|c|c|c|}
\hline & Peça & Máquina & Tempo & Quant/Máq \\
\hline & 1 & 5 & 0.416667 & 100 \\
\hline & 2 & 4 & 0.25 & 60 \\
\hline & 3 & 3 & 0.333333 & 80 \\
\hline & 4 & 2 & 0.208333 & 50 \\
\hline & 6 & 4 & 0.625 & 0 \\
\hline & 8 & 2 & 0.416667 & 150 \\
\hline & 9 & 1 & 0.083333 & 20 \\
\hline & & & 2.33333 & 460 \\
\hline
\end{tabular}

\begin{tabular}{|c|c|c|c|c|}
\hline Peça & $\begin{array}{c}\text { Quantidade } \\
\text { produzida }\end{array}$ & Estoque $\mathrm{t}=3$ & Demanda & Estoque $\mathrm{t}=4$ \\
\hline 1 & 100 & 0 & 100 & 0 \\
\hline 2 & 60 & 0 & 60 & 0 \\
\hline 3 & 80 & 0 & 80 & 0 \\
\hline 4 & 50 & 0 & 50 & 0 \\
\hline 5 & 0 & 30 & 30 & 0 \\
\hline 6 & 150 & 0 & 150 & 0 \\
\hline 7 & 0 & 0 & 0 & 0 \\
\hline 8 & 100 & 0 & 100 & 0 \\
\hline 9 & 20 & 0 & 20 & 0 \\
\hline 10 & 0 & 50 & 50 & 0 \\
\hline
\end{tabular}

Resolução do problema acima utilizando a Heuristica 1 e a programação das máquinas efetuada pelo modelo (2.31)-(2.35).

Valor Objetivo: 406.200

Período 1

Produção de peças no período 1 utilizando a liga 5

\begin{tabular}{|c|c|c|c|c|}
\hline & Peça & Máquina & Tempo & Quant/Maq \\
\hline & 5 & 1 & 0.016436 & 4 \\
\hline & 5 & 4 & 0.656539 & 126 \\
\hline & 7 & 2 & 0.325160 & 62.43072 \\
\hline & 7 & 3 & 0.656539 & 157.56936 \\
\hline & 10 & 1 & 0.640103 & 122.899776 \\
\hline & 10 & 2 & 0.331379 & 79.5396 \\
\hline & 10 & 5 & 0.656539 & 157.5693 \\
\hline
\end{tabular}




\begin{tabular}{|c|c|c|c|c|}
\hline$\infty$ & $00 \varepsilon$ & $09 \varepsilon$ & 0 & $\mathrm{OL}$ \\
\hline 0 & 0 & 0 & 0 & 6 \\
\hline 0 & 0 & 0 & 0 & 8 \\
\hline$O Z$ & $00 z$ & OZZ & 0 & $L$ \\
\hline Ol & 0 & 0 & Ol & 9 \\
\hline$O \varepsilon l$ & 0 & $O \varepsilon L$ & 0 & $\underline{s}$ \\
\hline Ob & 001 & 0 & $O b 1$ & $t$ \\
\hline 0 & Obl & 0 & OOL & $\varepsilon$ \\
\hline 0 & $O \rightarrow Z$ & 0 & $O b z$ & 2 \\
\hline 0 & 091 & 0 & 091 & 1 \\
\hline ; әnbols 3 & ерueшәव & $l=l$ ənbols 3 & әpep!queno & eీீ్d \\
\hline
\end{tabular}

\begin{tabular}{|c|c|c|c|c|}
\hline 069 & $9 Z \angle \varepsilon \angle 8^{\circ} \mathcal{E}$ & & & 1E701 \\
\hline $\mathrm{OL}$ & 0900 & $\downarrow$ & 9 & \\
\hline 8tt6sl & $\rightarrow Z \angle G \angle O 0^{\circ}$ & $z$ & $\downarrow$ & \\
\hline $2990 \diamond Z 1$ & StESLLO & $l$ & $\downarrow$ & \\
\hline $900 \angle 82$ & Eoset l.0 & $\varepsilon$ & $\varepsilon$ & \\
\hline 98662 レレ & $129969^{\circ} 0$ & $z$ & $\varepsilon$ & \\
\hline Z906.8६। & $9 Z 9669^{\circ} 0$ & $t$ & 2 & \\
\hline ZLt60 LOL & 乙†৪เ๕๐० & $\varepsilon$ & $z$ & \\
\hline Sal & S†્ESLIO & $\mathrm{s}$ & 1 & \\
\hline 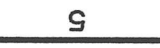 & $618050 \%$ & $t$ & 1 & \\
\hline bewnueno & odur 1 & eu!nbẹw & e5̃ d & \\
\hline
\end{tabular}

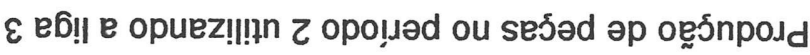

乙 opolı.dəd

\begin{tabular}{|c|c|c|c|}
\hline $09 \varepsilon$ & 0 & $09 \varepsilon$ & $\mathrm{Ol}$ \\
\hline 0 & 0 & 0 & 6 \\
\hline 0 & 0 & 0 & 8 \\
\hline Ozz & 0 & OZZ & $L$ \\
\hline 0 & 0 & 0 & 9 \\
\hline$\propto \varepsilon l$ & 0 & $O \varepsilon l$ & $s$ \\
\hline 0 & 0 & 0 & $\downarrow$ \\
\hline 0 & 0 & 0 & $\varepsilon$ \\
\hline 0 & 0 & 0 & 2 \\
\hline 0 & 0 & 0 & 1 \\
\hline$l=l$ anbols 3 & ерueuәg & $\begin{array}{l}\text { ep!̣nposd } \\
\text { epepplqueno }\end{array}$ & eకృ d \\
\hline
\end{tabular}




\section{Período 3}

Produção de peças no período 3 utilizando a liga 3

\begin{tabular}{|c|c|c|c|c|}
\hline & Peça & Máquina & Tempo & Quant/Máq \\
\hline & 1 & 4 & 0.235567 & 37.69072 \\
\hline & 1 & 5 & 0.811546 & 162.3092 \\
\hline & 2 & 4 & 0.60 & 120 \\
\hline & 3 & 2 & 0.494021 & 79.04336 \\
\hline & 3 & 3 & 0.835567 & 167.1134 \\
\hline & 3 & 5 & 0.024021 & 3.84336 \\
\hline & 4 & 2 & 0.30 & 160 \\
\hline & 8 & 1 & 0.135567 & 21.69072 \\
\hline & 8 & 2 & 0.041546 & 8.312 \\
\hline & 9 & 1 & 0.7 & 140 \\
\hline & & & 4.0625 & 800 \\
\hline
\end{tabular}

\begin{tabular}{|c|c|c|c|c|}
\hline Peça & Quantidade & Estoque $\mathrm{t}=2$ & Demanda & Estoque $\mathrm{t}=3$ \\
\hline 1 & 200 & 0 & 200 & 0 \\
\hline 2 & 120 & 0 & 120 & 0 \\
\hline 3 & 250 & 0 & 250 & 0 \\
\hline 4 & 60 & 40 & 100 & 0 \\
\hline 5 & 0 & 130 & 100 & 30 \\
\hline 6 & 0 & 10 & 10 & 0 \\
\hline 7 & 0 & 20 & 20 & 0 \\
\hline 8 & 30 & 0 & 30 & 0 \\
\hline 9 & 140 & 0 & 140 & 0 \\
\hline 10 & 0 & 60 & 10 & 50 \\
\hline
\end{tabular}

\section{Período 4}

Produção de peças no período 4 utilizando a liga 3

\begin{tabular}{|c|c|c|c|c|}
\hline & Peça & Máquina & Tempo & Quant/Máq \\
\hline & 1 & 5 & 0.416667 & 100 \\
\hline & 2 & 3 & 0.312500 & 60 \\
\hline & 3 & 2 & 0.235895 & 45.2984 \\
\hline & 3 & 3 & 0.065702 & 15.76848 \\
\hline & 3 & 5 & 0.098645 & 18.93984 \\
\hline & 4 & 2 & 0.208333 & 50 \\
\hline & 6 & 3 & 0.137110 & 26.32512 \\
\hline & 6 & 4 & 0.515312 & 123.67488 \\
\hline & 8 & 1 & 0.431978 & 82.939776 \\
\hline & 8 & 2 & 0.071084 & 17.06016 \\
\hline & 9 & 1 & 0.083333 & 20 \\
\hline & & & 2.576549 & 560 \\
\hline
\end{tabular}




\begin{tabular}{|c|c|c|c|c|}
\hline Peça & $\begin{array}{c}\text { Quantidade } \\
\text { produzida }\end{array}$ & Estoque $\mathrm{t}=3$ & Demanda & Estoque $\mathrm{t}=4$ \\
\hline 1 & 100 & 0 & 100 & 0 \\
\hline 2 & 60 & 0 & 60 & 0 \\
\hline 3 & 80 & 0 & 80 & 0 \\
\hline 4 & 50 & 0 & 50 & 0 \\
\hline 5 & 0 & 30 & 30 & 0 \\
\hline 6 & 150 & 0 & 150 & 0 \\
\hline 7 & 0 & 0 & 0 & 0 \\
\hline 8 & 1000 & 0 & 100 & 0 \\
\hline 9 & 20 & 0 & 20 & 0 \\
\hline 10 & 0 & 50 & 50 & 0 \\
\hline
\end{tabular}

Pode-se observar que o método heurístico não conseguiu a solução ótima, mas a solução encontrada pela heurística está em torno de $0.5 \%$ do valor ótimo.

Pode-se também notar que tanto na solução do problema linear inteiro misto como na solução do problema (2.26.)-(2.30) a utilização das máquinas está concentrada em algumas das máquinas e, na solução do problema (2.31)-(2.35) há uma utilização mais uniforme das máquinas, conforme esperava-se.

As ligas escolhidas para a produção das peças foram as mesmas, a menos do período 2, que no problema linear inteiro misto foi escolhida a liga 4 e na heurística 1 foi escolhida a liga 3. 


\section{BIBLIOGRAFIA}

[1] Afentakis P., Gavish B. (1986), Optimal Lot-sizing Algorithms for Complex Product Structures, Operations Research, v.34, n.2, 237-249.

[2] Afentakis P., Gavish B., Karmakar U. (1984), Computationally Efficient Optimal Solutions to the Lot-Sizing Problem in Multistage Assembly Systems, Management Science, v.30, n.2, 222-239.

[3] Bazaraa, M. S., Jarvis J. J. (1977), Linear Programming and Network Flows John Wiley \& Sons.

[4] Bazaraa, M.S., Sherali, H.D. (1981), On the Choice of Step Size in Subgradient Optimization, European Journal of Operational Research 7, 380-388.

[5] Bahl, H. C., Ritzman, L. P., Gupta, J. N. D., (1987), Determining Lot Sizes and Resource Requirements : A Review, Operations Research, v.35, n.3, 329-345.

[6] Baker, K. R. (1993), Requirements Planning, em Graves, S.C., Kan, A. H. G. Rinnooy, Zipkin, P. H. Logistics of Production and inventory, Handbooks in operations Research and management science: Elsevier Science Publishers B. v.4, $571-627$.

[7] Berreta, R. E., Armentano, V. A., A Heuristic for Lot-sizing in Multi-Stage Systems, Alaska.

[8] Berreta, R. E., Armentáno, V. A. (1996), Relatório da Fapesp, $1^{\circ}$ semestre.

[9] Billington P. J., McClain J. O., Thomas L. J. (1983), Mathematical Programming Approaches to Capacity MRP Systems: Review, Formulation and Problem Reduction, Management Science, V. 29, n. 10, 1126-1141.

[10] Billington P. J., McClain J. O., Thomas L. J. (1986), Heuristics for multilevel LotSizing With a Bottleneck, Management Science, v. 32, n. 8, 989-1006. 
[11] Billington P. J., Blackburn J., Maes J., Millen R., Wassenhove L. N. V. (1995), Multi-Item Lotsizing Capacited Multi-Stage Serial Systems, IIE Transactions, v.26, n.2, 12-18.

[12] Blackburn, J. D., Millen, R.A. (1982), Improved Heuristics for Multistage Requirements Planning Systems, Management Science, v.28, n.1, 44-56.

[13] Burbidge, J. L. (1981), Planejamento e Controle da Produção, Editora Atlas, São Paulo.

[14] Chiu H. N., Lin T. M. (1988), An Optimal Lot-sizing Model for Multi-Stage Series/Assembly Systems, Computers and Operations Research, V.15, n.5 403-415.

[15] Clark, A. R., Armentano, V. A. (1995), A Heuristic for a Resource-Capacitated Multi-Stage Lot-Sizing Problem with Lead Times, Journal of the Operational Research Society, v.46, n.10, 1208-1222.

[16] Dantzig, George (1955), Optimal Solution to a Dynamic Leontief Model With Substitution, Econométrica, v.23, 295-302.

[17] Diaby, M., Bahl H., Karwan, M. H., Ziont, S. (1992), Capacitated Lot-Sizing and Scheduling by Lagrangean Relaxation, European Journal Of Operational Research, n. 59, 444-458.

[18] Dixon, P.S., Silver, E.A. (1981), A Heuristc Solution procedure for the Multi Item, Single Level, Limited Capacity, Lot-sizing Problem, Journal of Operations Management, v.2, n.1, 233-39.

[19] Fernandes, F. C. F. (1991) Concepção de um Sistema de Controle da Produção para uma Manufatura Celular, (Tese de Doutorado - EESC/USP).

[20] Florian M., Lenstra J. K., Rinnoy Kan, A. H. G. (1980), Deterministic Production Planning Algorithms and Complexity, Management Science, v.26, n.7, 669-679.

[21] Graves, S. C. (1981), A review of Production Scheduling, Oper. Res, v.29 n.4, 646-675.

[22] Graves, S.C., Kan, A. H. G. Rinnooy, Zipkin, P. H. (1993), Logistic of production and inventory (Handbooks in operations research and management science: $v$. 4), Elsevier Science Publishers B. V.

[23] Kuik, R., Salomom, M., Van Wassenhiose, L. N., Maes, J. (1993), Linear Programming, Simulated Annealing and Tabu Search Heuristics for Lotsizing in Bottleneck Assembly Systems, IIE Transactions, v.25, n.1, 62-72. 
[24] Kuik, R., Salomon, M. (1990), The Multi-Level Lotsizing Porblem: Evaluation of a Stochastic Optimization Heuristic, European Journal of Operational Research,v.45,25-37.

[25] Lambrecht, M., Vanderveken, H., (1979), Heuristic Procedures for the Single Operation Multi Item Loading Problem, AllE Transactions, v. 11 n.4, 319-326.

[26] Lasdon, L. S. (1970), Optimization Theory for Large Systems, Macmillan Publishing Company, New York.

[27] Luenberger, D. G. (1984), Introduction to Linear and Non-Linear Programming, Second Edition, Addison-Wesley, Reading, Mass.

[28] Maes J., McClain, J. O., Van Wassenhove, L. N. (1991), Multilevel Capacitated Lotsizing Complexity and LP Based Heuristic, European Journal of Operational. Research, v.53, 131-148.

[29] Maes, J., and Van Wassenhose, L.N. (1986), A Simple Heuristic for the Multiitem Single

Level Capacitated Lot Sizing Problem, Operations Research Letters, v.4, 265274.

[30] McClain, J. O., Thomas, L. J., Weiss, R. N., (1989), Efficient Solutions to a Linear Programming Model for Production Scheduling With Capacitated Constraints, IIE Transactions, v. 21, n. 2, 144-152.

[31] Nemhauser, G. L., Wolsey, L. A. (1988), Integer and Combinatorial Optimization, Wiley-Interscience Publication.

[32] Resende, M. O. (1992), Princípios de processos de produção v.1, Escola de Engenharia de de São Carlos, Universidade de São Paulo, publicação 016/92.

[33 Roll, Y., Karni, R. (1991), Multi-ltem, Multi-Level Lot Sizing With an Aggregate Capacity Constraint, European Journal of Operational Reserch, v.51, 73-87.

[34] Shapiro, J. (1993), Mathematical Programming Models and Methods for Production Planning and Scheduling, em Graves, S.C., Kan, A. H. G. Rinnooy, Zipkin, P. H. Logistics of Production and inventory, Handbooks in operations Research and management science: Elsevier Science Publishers B. v.4, 371 443.

[35] Santos, M. O. (1996), Dimensionamento de Lotes Multiestágios Capacitado: Um novo Modelo e Proposta de Solução, Dissertação de Mestrado do ICMSCUSP. 
[36] Santos, M. O., Meza, E. S., Arenales, M. N., (1996), Dimensionamento de Lotes Multiestágios Capacitado: Um novo Modelo e Método de Solução, Anais do XXVIII SBPO e VIII CLAIO, 1074-1079.

[37] Toledo, F. M. B., Armentano, V. A., França, P. M. (1994), Um Método Ótimo Para o Problema de Dimensionamento de lotes com restrições de Capacidade, SBPO.

[38] Trigeiro, W. W., Thomas, L. J., McClain J. O. (1989), Capacitated Lot Sizing With Setup Times, Management Science, v.35, n. 3, 353-366.

[39] Wagner, H. M. (1986) - Principles of Operations Research - Prentice-Hall, Inc.

[40] Wagner, H. M. e Whitin, T. M. (1958), Dynamic Version of the Economic Lot Size Model, Management Science, v.5, n.1, 89-96.

[41] Veinott, A. F. Jr. (1969), Minimum Concave-Cost Solution of Leontief Substitution Models of Multi-facility Inventory Systems, Operations Research, march-april, 262-291.

[42] Vianna, A. C. G., Arenales, M. (1995), Problema de Programação da Produção numa Fundação Automatiizada, Gestão \& Produção, v.2, 244-263. 